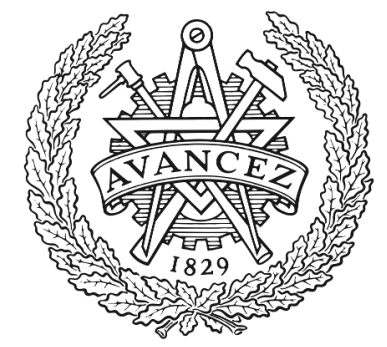

CHALMERS

UNIVERSITY OF TECHNOLOGY

\title{
Calculation of optical properties of light-absorbing carbon with weakly absorbing coating: A model with tunable transition from film-coating to
}

Downloaded from: https://research.chalmers.se, 2023-04-26 10:10 UTC

Citation for the original published paper (version of record):

Kanngiesser, F., Kahnert, M. (2018). Calculation of optical properties of light-absorbing carbon with weakly absorbing coating: A model with tunable transition from film-coating to spherical-shell coating. Journal of Quantitative Spectroscopy and Radiative Transfer, 216: 17-36. http://dx.doi.org/10.1016/j.jqsrt.2018.05.014

N.B. When citing this work, cite the original published paper. 


\title{
Calculation of optical properties of light-absorbing carbon with weakly absorbing coating: a model with tunable transition from film-coating to spherical-shell coating
}

\author{
Franz Kanngießer ${ }^{\mathrm{a}}$, Michael Kahnert ${ }^{\mathrm{a}, \mathrm{b}}$ \\ ${ }^{a}$ Department of Space, Earth and Environment, Chalmers University of Technology, SE-412 \\ 96 Gothenburg, Sweden \\ ${ }^{b}$ Research Department, Swedish Meteorological and Hydrological Institute, Folkborgsvägen \\ 17, SE-60176 Norrköping, Sweden
}

\begin{abstract}
Optical properties of particles consisting of light-absorbing carbon (or soot) and a weakly absorbing coating material are computed at a wavelength of $355 \mathrm{~nm}$ and $532 \mathrm{~nm}$. A morphological particle models is used, in which small amounts of coating are applied as a thin film to the surface of the aggregate, while heavily coated aggregates are enclosed in a spherical shell. As the amount of coating material is increased, a gradual transition from film-coating to spherical-shell coating is accounted for. The speed of this transition can be varied by specifying a single parameter. Two different choices of this parameter, corresponding to a slow and a rapid transition from film-coating to spherical-shell coating, respectively, are investigated. For low soot volume fractions the impact of this transition on the linear depolarisation ratio $\delta_{l}$ is most pronounced. The model that describes a rapid transition to a spherical coating yields results for $\delta_{l}$ that are more consistent with existing lidar field measurements than the slow-transition model. At $532 \mathrm{~nm}$ the relative uncertainty in modelled $\delta_{l}$ for a rapid transition values due to uncertainties in the aggregate's geometry and chemical composition are estimated to range from 109 to $243 \%$, depending on the soot volume
\end{abstract}

Email addresses: franz.kanngiesser@chalmers.se (Franz Kanngießer), michael.kahnert@smhi.se (Michael Kahnert)

Preprint submitted to J. Quant. Spectrosc. Radiat. Transfer 
fraction. At $355 \mathrm{~nm}$ the relative uncertainties were estimated to range from 90.9 to $200 \%$.

Keywords: Scattering, Aerosol, Soot, Depolarization ratio

2010 MSC: 00-01, 99-00

\section{Introduction}

Particles consisting of light-absorbing carbon or soot are among the atmospheric aerosol particles with the largest impact on the Earth's climate. They can influence the climate system through their role in cloud formation processes,

5 by reducing the albedo of snow and ice in polar and mountainous regions, or by direct absorption of solar radiation [1, 2]. Also, soot particles have an adverse impact on air quality and human health [3]. Observations of soot particles by remote sensing techniques play an important role in monitoring sources, transport pathways, and deposition of soot particles in the atmosphere. These observations are also essential for constraining and improving aerosol transport and air quality forecasting models. The interpretation of remote sensing observations as well as predictions of the climate impact of soot particles relies on a thorough understanding of the particles' optical properties.

Soot particles themselves consist of highly absorbing carbonaceous material.

15 Aged atmospheric soot particles are commonly coated by weakly absorbing material, which can complicate the modelling of optical properties of soot particles [4. Both images of atmospheric soot particles and chemical analyses often indicate a thick coating and hence a low soot volume fraction [5, 6, 7, 8, In [8] $50 \%$ of freshly emitted soot particles were reported to be heavily coated, thus

20 indicating a fast coating process in the atmosphere. As shown in [7] the coating itself may consist of different materials.

Various models have been employed to investigate the impact of the particles' morphological features on radiative and optical properties. The extent to which morphological features of coated soot particles need to be resolved in models depends on the intended application. To account for the coating different 
particle models have been used. The climate relevant impact on the broadband solar radiative flux can be investigated by assuming a spherical core-shell or the recently introduced core grey shell model [9]. Using morphologically more complex particle models requires more computational efforts. The impact of the use of simplified particle models in climate models can be quantified by comparing the results obtained for simplified models to those obtained for more complex models (e.g. [10]).

Prior work on the uncertainty introduced into climate models by assuming strongly simplified shapes of soot particles focused on radiative properties, such as the mass absorption cross section (e.g. [11]) or the scattering and absorption cross sections [12, 13, as well as on the single scattering albedo and asymmetry parameter. The closed cell or concentric core-shell monomer model employed by [14, 15, 13] uses aggregates where each individual monomer is coated by a spherical shell. Other approaches apply the coating onto the aggregate of 40 contacting soot monomers. Two other approaches in which the coating follows the shape of the aggregate were used in 13 . The first approach defines a fixed coating thickness. A coating layer with this thickness is then added to the aggregate. The other approach adds small coating volumes at the edges of the soot aggregate or the coating until a prescribed volume fraction is reached. In 45 another study coating was added by first filling voids in branches with more densely packed monomers; then the coating is continued in descending order of the density of monomer packing [12. Partial soot inclusions in spherical coating shells were investigated in [12,16]. Both the model which filled voids in branches and the partial soot inclusion model discussed in 12 served as a references for comparison with simpler core-shell and homogeneous sphere models. However there were differences between the two morphologically more complex models. The scattering cross section is larger if the surface of coated soot increases. In [16] the impact of monomer surfaces intersecting with the coating surface was investigated. The difference between the two models was less than $5 \%$ for optical cross sections, asymmetry factor and single scattering albedo; therefore, this morphological feature may be ignored. 
A numerical investigation presented in [17] was carried out to evaluate the possibility of determining the primary particle size of bare soot aggregates by measuring the depolarisation of light. In that numerical investigation it was found that refractive index, the aggregate shape and connections of the monomers making up the aggregate have a strong impact on the depolarisation.

Compared to climate models, remote sensing applications may require morphologically more complex models. One quantity frequently used in lidar remote sensing is the linear depolarisation ratio, $\delta_{l}$. This quantity is a key element in classifying aerosol species using lidar techniques. The aerosol classification schemes of the Cloud-Aerosol Lidar and Infrared Pathfinder Satellite Observations (CALIPSO) [18] and for the planned Earth Clouds, Aerosols and Radiation Explorer (EarthCARE) [19, 20, rely on measurements of $\delta_{1}$. Depolarisation is highly sensitive to morphology, and even to particle inhomogeneity [21]. It is therefore expected that a correct description of depolarisation requires significantly more realistic model particles than radiative forcing computations in climate models.

Images of coated soot particles presented by [6, 22] suggest that the coating in case of low soot volume fraction tends to form a spherical or almost spherical shape, which encapsulates parts of the soot aggregate, while parts of the aggregate are sticking out of the coating. A corresponding model has been considered in, e.g., 9, 16]. However, this model is not expected to be very realistic for high soot volume fractions (i.e., thin coatings). For those the coating forms a thin film that closely follows the shape of the aggregate, similar to the model in 13. It is plausible to assume that there should be a smooth transition from the nonspherical to the spherical coating, which would take place at intermediate soot volume fractions. Also, as soot particles age in the atmosphere, their fractal dimension tends to increase, resulting in a collapse and compaction of the aggregate structure [4, 23, 24, 25]. In [8] the particles with the lowest soot volume fraction were reported to have the highest fractal dimension, i.e. being the most compact particles. Particles with higher soot volume fractions were reported to have lower fractal dimension. 
Thus, most coating models considered earlier can be expected to be realistic either for low or for high soot volume fractions. A recent study presented a first attempt to cover the entire range of volume fractions by devising a coated aggregate model that accounts for the transition from thinly coating films to spherical coatings [26]. However, the fractal dimension has been assumed to be constant, i.e., the transition from lacy to more compact soot aggregates during the coating process has been neglected. The computational results were compared to those obtained with the closed cell model. The cross sections of both models were in relatively good agreement for high soot volume fractions (i.e., thin coatings). However, the depolarisation ratios were found to differ between the two models. The depolarisation ratios of soot particles obtained with the coated aggregate model were more consistent with field observations than those obtained in the closed cell model. However, there seems to be a certain risk that the coated aggregate model overestimates depolarisation at low volume fractions and for large particle sizes. This suggests that the model particles proposed in 26] might be too nonspherical for low soot volume fractions.

To lower the depolarisation by soot particles one could make the transition from thinly-coating films to spherical coatings more rapid, because this will make particles with low soot volume fractions more spherical. This can be achieved by (i) reducing the radius of the critical sphere defined in [26] that marks the transition from film-coating to spherical coating; and (ii) assuming that the fractal dimension of the aggregate increases with decreasing soot volume fraction (i.e., increased coating thickness).

We hypothesize that the speed of transition from film-coating to spherically encapsulated soot aggregates is an essential morphological parameter to which the linear depolarisation ratio is highly sensitive. By a high speed of transition, we mean that the coating becomes spherical at relatively small amounts of coat115 ing material (i.e, at relatively high soot volume fractions); while a low speed of transition requires higher amounts of coating material before the shell becomes spherical.

We will test our hypothesis by formulating a particle model in which the 
speed of transition can be continuously adjusted by tuning a single parameter. We will then compare two choices of this parameter, which simulate a slow and a rapid transition, respectively, from film-coating to spherical coating as more liquid-phase material is added to the soot aggregate. The computational results for the linear depolarisation ratio are gauged against published observations from field measurements.

A more detailed description of the particle model, as well as a description of the computational methods are given in Sec. 2. The results are presented and discussed in Secs. 3 and 4 , respectively. Concluding remarks are given in Sec. 5.

\section{Methods}

\subsection{Particle models}

Atmospheric soot aerosol particles can be approximated as aggregates consisting of spherical monomers following the scaling relation [27]:

$$
N=k_{0}\left(\frac{R_{g}}{a}\right)^{D_{f}}
$$

In Eq. (1) $N$ denotes the number of monomers, $a$ the monomer radius, $k_{0}$ is the fractal prefactor and $D_{f}$ the fractal dimension. The fractal dimension takes values between 1 (linear aligned monomers) and 3 (spheres). The radius of gyration $R_{g}$ is given by

$$
R_{g}=\sqrt{\frac{1}{N} \sum_{i=1}^{N}\left|\vec{r}_{i}-\vec{r}_{c}\right|^{2}}
$$

Here $\vec{r}_{i}$ is the position vector of the centre of the $i$ th monomer and $\vec{r}_{c}$ the position vector of the aggregate's centre of mass.

In this study aggregates following the scaling relation in Eq. (1) have been created using the cluster-cluster aggregation algorithm presented in [28].

This method mimics aggregate formation processes in nature more closely than 
diffusion-limited aggregation algorithms. The cluster-cluster algorithm combines smaller aggregates, each fulfilling the scaling relation, to larger aggregates, while a diffusion limited algorithm adds one monomer at a time.

We generated aggregates consisting of 8, 64, 216 and 512 monomers. For all particles a prefactor of $k_{0}=0.7$ was assumed following the measured median reported in [29]. For each size, five different stochastic realisations of aggregates with prescribed fractal parameters have been generated. These will be used to estimate the variation of optical properties with changes in the aggregate geometry.

Based on the conclusions in [26], we hypothesise that a crucial morphological feature of coated soot particles is the transition from a coating film of liquid material that closely follows the shape of the aggregate to a spherical coating that encapsulates the aggregate. More specifically, we hypothesise that the linear depolarisation ratio is highly sensitive to the speed of this transition, i.e., to whether the onset of sphericity occurs at relatively low or high amounts of coating.

To test this hypothesis, we devise a model in which the coating is added to the aggregate layer by layer, where the layer thickness $d$ is equal to the volume cell size (or dipole spacing) that we employ in the discrete dipole approximation ${ }_{155}$ (DDA, see section 2.3). More specifically, we proceed as follows. For each aggregate the maximum dimension $\mathrm{D}$ is determined. Based on this maximum dimension we define a critical sphere of Diameter $D_{\text {c }}$ centred at the aggregate's centre of mass. Inside the critical sphere the coating is applied layer by layer following the shape of the aggregate, while being constrained not to be applied outside the critical sphere. These successive coating layers are represented by the grey lines in Fig. 1 . Once the critical sphere is completely filled, successive layers of coating are applied radially onto the sphere (see dash-dotted grey sphere in Fig. 11. The whole process is terminated once the desired soot volume fraction is reached.

The critical diameter $D_{\mathrm{c}}$ is a tunable parameter of the model. We consider two choices of this parameter. In the first one (the "slow" transition model), 
we use $D_{\text {c,slow }}=D+2 d$. Thus, the critical sphere completely encapsulates the aggregate; its diameter is larger by $2 d$ than that of the smallest circumscribing sphere (see solid blue sphere in Fig. 1). In the second one (the "rapid" transition model), we use $D_{\text {c,rapid }}=0.6(D+2 d)$. Thus, the critical sphere does not completely encapsulate the aggregate (see dashed blue sphere in Fig. 11) 11

Thus the rapid-transition model simulates a coating process where the transition from film-coating to spherical coating occurs rather rapidly as more coating material is added. In the slow-transition model, the onset of sphericity is reached at a much later stage at lower soot volume fractions, i.e., when a larger amount of coating has been added to the aggregate. In the slow-transition model, sphericity is not reached before the aggregate is completely encapsulated. In the rapid-transition model, the coating becomes spherical at higher soot volume fractions, where part of the aggregate is allowed to stick out of the coating. This is consistent with images of coated soot aggregates (e.g. [30]).

The rapid-transition model is further motivated by recent laboratory measurements reported in 31, where the morphological changes of soot particles during the process of condensation of coating material are investigated. They observed that a gradual increase of the amount of coating material first had a negligible effect on the particles' mobility diameter, followed by a sudden sharp increase in diameter. This behaviour is explained as follows. At first, inner voids of the aggregates are filled with the coating material. Once the open voids are filled, the particles grow radially in diameter. The mechanism of soot particle growth due to condensation of matter presented in 31 qualitatively supports 190 our assumption of a transition from a coating film to growth of an encapsulating sphere.

As stated in section 1 restructuring of the aggregate with decreasing soot volume fraction was assumed for both the slow and the rapid transition model

\footnotetext{
${ }^{1}$ We emphasize that the terms slow and rapid transition models are employed for the sake of brevity. However, both are, in fact, based on one a single model with two different choices of the free parameter in the model.
} 
based on $4,23,8,25,24$. The process of restructuring of the soot aggregate during the process of coating is rather complex and depends on the coating material. For sulphuric acid a proportional increase in compactness with increasing coating mass was reported in [4]. However for oleic acid a rapid compaction was reported for small amounts of coating material added to the aggregate. After that rapid collapse of the aggregate further condensation of coating material onto the aggregate led to further compaction, but at a smaller rate [23, 24]. The condensation of aromatic hydrocarbons leads to a restructuring of the aggregate after a certain threshold of condensated mass is passed. After reaching that threshold the compactness increases with increasing amount of coating material [25]. The compactness increases with condensation of coating material until the maximum compactness of the aggregate is reached [23, 24, 25]. Additionally [8] reported that atmospheric soot particles with the thickest coating had the highest fractal dimension (i.e. compactness).

To reflect the restructuring an increase in the fractal dimension $D_{f}$ from 2.0 to 2.6 was included in our model. The assumed relation between soot volume fraction $f_{\text {vol }}$ and fractal dimension $D_{f}$ for this study is given in Tab. 1 As a simplification, changes in fractal dimension were not adapted for different coating materials. The values of $D_{f}$ were based on [29]. In that study they measured the fractal dimension using three-dimensional scans, which systematically yield higher results than methods inferring the fractal dimension from two-dimensional images.

To illustrate the effect of the fractal dimension on the compactness of aggregates two examples of aggregates with the same number of aggregates $(N=64)$ and fractal prefactor $\left(k_{0}=0.7\right)$ are depicted in Fig. 2. Figure 2a shows an aggregate with a fractal dimension of $D_{f}=2.0$, while Fig. $2 \mathrm{~b}$ shows an aggregate with a fractal dimension of $D_{f}=2.6$. The latter is more compact than the former.

Several examples of soot particles used for the calculations with $N=64$ are shown in Fig. 3. The aggregates are plotted in grey, while the coating is plotted in yellow. The top row $(\mathrm{a}-\mathrm{c})$ shows model particles based on the slow- 


\begin{tabular}{cc}
$f_{\text {vol }}(\%)$ & $D_{f}$ \\
\hline 100 & 2.0 \\
75 & 2.0 \\
50 & 2.2 \\
25 & 2.4 \\
10 & 2.6
\end{tabular}

Table 1: Assumed relation between the soot volume fraction $f_{\mathrm{vol}}$ and the fractal dimension $D_{f}$

transition mdoel, while the bottom row ( $\mathrm{d}-\mathrm{f}$ ) shows corresponding particles based on the rapid-transition model. The three columns show examples of model particles with volume fractions and fractal dimensions of $f_{\mathrm{vol}}=50 \%$ and $D_{f}=2.2$ (left, panels a and d), $f_{\text {vol }}=25 \%$ and $D_{f}=2.4$ (centre, panels b and e), and $f_{\mathrm{vol}}=10 \%$ and $D_{f}=2.6$ (right, panels c and f). This example illustrates the increasing sphericity of the coated aggregates with decreasing soot volume fraction, as well as the differences between the slow- and rapidtransistion models.

The calculations were carried out for two wavelengths, $\lambda=355 \mathrm{~nm}$ and $532 \mathrm{~nm}$. These are the third and second harmonics of the neodymium-doped yttrium aluminium garnet (Nd:YAG) laser, which is commonly employed in lidar instruments (e.g. [32, 33, 34, 35, 20, 36, 37, 38, 39; ; see also references in [26]). At those two wavelengths the slow-transition and rapid-transition models are compared to each other assuming point-contacting monomers.

The refractive index $m=n+i k$ for soot at $355 \mathrm{~nm}$ was determined with Eqs. (3) and (4) [40] with $\lambda$ being the wavelength in $\mu \mathrm{m}$. The expression has been obtained by fitting measurements on soot aggregates in the wavelength range between $0.4 \leq \lambda \leq 30 \mu \mathrm{m}$. Therefore, the values for the real and imaginary part 
of the refractive index at $0.355 \mu \mathrm{m}$ had to be obtained by extrapolation. ${ }^{2}$

$$
\begin{aligned}
& n=1.811+0.1263 \ln \lambda+0.027 \ln ^{2} \lambda+0.0417 \ln ^{3} \lambda \\
& k=0.5821+0.1213 \ln \lambda+0.2309 \ln ^{2} \lambda-0.01 \ln ^{3} \lambda
\end{aligned}
$$

This resulted in a refractive index of $m_{\text {soot }}=1.66284+i 0.71528$ for $\lambda=$

$0.355 \mu \mathrm{m}$. For the refractive index at $532 \mathrm{~nm}$ we use $m_{\text {soot }}=1.76+i 0.63 \mathrm{in}$ accordance with the void-fraction-curve discussed in [1]. The coating material is assumed to be sulphate, which has a refractive index of $m_{\mathrm{SO}_{4}}=1.43+i 10^{-8}$ for $532 \mathrm{~nm}$ and $m_{\mathrm{SO}_{4}}=1.45+i 10^{-8}$ for $355 \mathrm{~nm}$. The refractive indices for sulphate were taken from the OPAC (optical properties of aerosols and clouds) software package (Version 4.0b). 41].

\subsection{Approach to estimate uncertainties}

We estimate the model uncertainties by considering seven sources of error, namely, uncertainties in aggregate geometry, uncertainties in fractal prefactor, fractal dimension, monomer radius, overlapping of spheres, uncertainties in the refractive index of soot, and uncertainties in the refractive index of the coating material.

- As mentioned earlier, we perform computations for different stochastic realisations of aggregates with prescribed fractal parameters. This allows us to assess errors introduced by representing a large ensemble of aggregates by a small selection of (more or less) representative geometries.

- Based on 29] we assumed a fractal prefactor of $k_{0}=0.7$ in our reference calculation. The fractal prefactors of the samples analysed in 29] ranged from $k_{0}=0.25$ to $k_{0}=1.6$. We performed additional calculations with these values but assumed a constant fractal dimension of the aggregates.

\footnotetext{
${ }^{2}$ It turns out that the polynomials in $\sqrt{3}$ and 4 do not vary very rapidly in the range from 355 to $400 \mathrm{~nm}$. However, we did perform estimates of the error introduced by the extrapolation assumption. The approach to the error analysis is described in the the next section.
} 
- To estimate the uncertainty of the model imposed by variations in fractal dimensions additional calculations were carried out assuming variations in fractal dimension of $\Delta D_{f}= \pm 0.2$ compared to the value tabulated in Tab. 1 .

- Different values of the monomer radius have been reported ranging from $a=10 \mathrm{~nm}$ [1] to $a=28 \mathrm{~nm}$ [8]. In our reference calculations we assumed a monomer radius of $a=25 \mathrm{~nm}$. The influence of changes in monomer radius were investigated in two different ways: a) assuming unchanged number of monomers and thus a change in the overall aggregate size and b) adapting the number of monomers to the changed monomer radius and thus having a constant overall aggregate size.

- In the reference computations point-contacting monomers are assumed. This is an idealised shape. Soot aggregates observed in nature typically consist of overlapping monomers [29, 42, 43]. The effect of adding overlapping in the model is investigated. To parametrize the overlap between monomers the overlapping factor $C_{o v}$ defined in Eq. (5) was used. Let $d_{p}=2 a$ be the diameter of the monomer and let us denote by $d_{i j}$ the distance between the centres of the two neighbouring monomers $i$ and $j$. Then the overlapping factor $C_{o v}$ is defined by [4]

$$
C_{o v}=\frac{d_{p}-d_{i j}}{d_{p}}
$$

We have $C_{o v}=0$ if two neighbouring monomers are in point-contact, and $C_{o v}=1$ if the the monomers completely overlap. Thus the overlapping factor describes the fraction by which the radius of a monomer intersects with its neighbouring monomers. As a simplification it was assumed, that the overlapping factor within an aggregate does not change. To apply the overlapping on the previously created aggregates the coordinates of each monomer's centre where multiplied by $\left(1-C_{o v}\right)$ following the approach described in 43 . As the volume equivalent radius of the aggregate, which is used as an input parameter for the scattering calculations, 
is fixed this results in an increase of the monomers' radii. Figure 4 shows two aggregates composed of $N=8$ monomers, one aggregate consisting of point-contacting monomers (Fig. 4a) and the other aggregate consisting of overlapping monomers with an overlapping factor of $C_{o v}=0.15$ (Fig. $4 \mathrm{~b})$.

- Soot consists of amorphous carbonaceous material with microphysical and dielectric properties that can depend on the combustion conditions under which the soot is being produced. The electronic structure of soot has been described as a mixture of $s p^{2}$ and $s p^{3}$ orbitals. Further, soot can contain a variable amount of void fractions. Accordingly, measurements can constrain the refractive index of soot only to a certain range (see the review in [1]). This is a potentially important source of uncertainty in model calculations. Here, we quantify the range of uncertainty by performing computations for two values of the refractive index of soot. The first one is our reference value at $532 \mathrm{~nm}$ of $m_{\mathrm{soot}, 1}=1.76+i 0.63$, which lies close to the lowest end of the void-fraction curve discussed in [1]. The second one is $m_{\mathrm{soot}, 2}=1.95+i 0.79$, which lies at the upper end of the void-fraction curve. Following the review in 1, we assume that these two values brace the range of refractive indices that are likely to be encountered in the majority of atmospheric soot aerosols. Analogously we chose two different refractive indices of soot for $355 \mathrm{~nm}$. The first one is our reference value for $355 \mathrm{~nm}: m_{\mathrm{soot}, 3}=1.66284+i 0.71528$, obtained by extrapolating Eqs. 3 and 4 and $m_{\text {soot }, 4}=1.68586+i 0.67251$ which was obtained by using the results of Eqs. 3 and 4 for $400 \mathrm{~nm}$ and assuming no spectral dependence of the refractive index of soot for UV wavelengths.

- The coating material can be a mixture of different compounds, such as sulphate and organic substances. The chemical composition determines the refractive index; hence the refractive index of the coating material can be another source of uncertainty. For our reference computations, we assumed that the coating consists of pure sulphate with a refractive 
index at $532 \mathrm{~nm}$ of $m_{\mathrm{SO}_{4}}=1.43+i 10^{-8}$. In addition, we performed computations for a coating consisting of organic material with a refractive index of $m_{\text {organic }}=1.53+i 0.0055$ [45]. As for $532 \mathrm{~nm}$ we assumed for our reference calculations for $355 \mathrm{~nm}$ a coating material consisting of pure sulphate with a refractive index of $m_{\mathrm{SO}_{4}}=1.45+i 10^{-8}$. Additional computations were performed for pure toluene coating with a refractive index for $355 \mathrm{~nm}$ of $m_{\text {toluene }}=1.632+i 0.047$ [46]. These refractive indices of pure non-absorbing sulphate and pure mildly absorbing organic coating act as estimates for the lower and upper bounds of the refractive index of the coating material.

To keep computation times within reasonable limits the calculations for different fractal dimension, different refractive indices and different monomer radii with changing number of monomers were performed for a single particle geometry per aggregate size and soot volume fraction. The uncertainty estimates have been performed for the rapid transition model at both wavelengths of $532 \mathrm{~nm}$ and $355 \mathrm{~nm}$.

\subsection{Discrete dipole approximation}

Single scattering properties of the soot particles were calculated using the DDA. This method is based on solving the volume-integral equation of electromagnetic scattering. The volume integral is discretised by dividing the scatterer into $n$ polarisable volume cells (or dipoles) much smaller than the wavelength. This leads to a system of linear equations that can be solved by standard numerical techniques. The DDA allows for scatterers to have arbitrary shapes. Here the publicly available DDA code ADDA (Version 1.2) has been used [47. A brief introduction into the theoretical foundation of the DDA can be found 335 in [48]. A more detailed account of the method can be found in [49, 50].

The accuracy of the DDA for computing optical properties of soot aggregates has been investigated earlier [51] by comparison with the superposition T-matrix method, and by using the reciprocity condition [52. Here we used a dipole 
spacing $d$ such that $|m| k d \leq 0.358$, where $m$ is the refractive index of soot, and where $k$ denotes the wavenumber of light in vacuum.

In our case, the number of dipoles $n=n_{\text {soot }}+n_{\text {coating }}$ can be partitioned into $n_{\text {soot }}$ volume cells of soot and $n_{\text {coating }}$ volume cells of coating. Then the soot volume fraction is given by $f_{\text {vol }}=n_{\text {soot }} /\left(n_{\text {soot }}+n_{\text {coating }}\right)$.

To control the target size the volume-equivalent radius $r_{\text {eff }}$ is used in ADDA. For bare aggregates consisting of $N$ point-contacting monomers of radius $a$ the volume equivalent radius $r_{\text {eff, agg }}$ is calculated using:

$$
r_{\mathrm{eff}, \mathrm{agg}}=a N^{\frac{1}{3}}
$$

The volume equivalent radius $r_{\text {eff }}$ for the coated aggregate is then calculated by

$$
r_{\mathrm{eff}}=\frac{r_{\mathrm{eff}, \mathrm{agg}}}{f_{\mathrm{vol}}^{\frac{1}{3}}}
$$

ADDA gives the complete scattering matrix as well as the extinction and absorption cross sections and the corresponding efficiencies for the scatterers. From the scattering matrix we can compute other optical parameters of interest, such as the linear backscattering depolarisation ratio 53

$$
\delta_{l}=\left.\frac{S_{11}-S_{22}}{S_{11}+S_{22}}\right|_{\vartheta=180^{\circ}}
$$

$S_{11}$ and $S_{22}$ are the 11 and 22 element of the scattering matrix in the backscattering direction $\left(\vartheta=180^{\circ}\right)$. For particles with spherical symmetry the backscattering depolarisation ratio is zero. Thus the depolarisation ratio is sensitive to changes in particle shape [53, 54].

Within ADDA the orientation averaging of the targets is performed numerically over discrete orientations [4]. For each scatterer 1024 orientations were used.

\section{Results}

\subsection{Visible light}

Figure 5 shows the scattering and the absorption cross section for the slowtransition and the rapid-transition model as well as the ratio of the optical cross 
index for the soot and $m_{\mathrm{SO}_{4}}=1.43+i 10^{-8}$ as refractive index of the coating material.

The differences between the calculated scattering and absorption cross sections are relatively small. In case of $f_{\mathrm{vol}}=100 \%$ the results are identical, as they should. The ratio of the optical cross sections of the rapid-transition to the slow-transition model is close to unity, indicating that both coating models yield similar optical cross sections. In Fig. 5, as for all following figures, the size of the coated aggregates is expressed by the volume equivalent radius $a_{\mathrm{eff}}$ of a spherical particle having the same volume as the aggregate. soot volume fraction. Decreasing the soot volume fraction and thereby adding coating material results in particle growth. An increase in particle size enlarges the geometric cross section, which generally increases the optical cross sections. The enhancement due to coating can be quantified by calculating the ratio 370 $C_{\mathrm{abs}}\left(f_{\mathrm{vol}}<100 \%\right) / C_{\mathrm{abs}}\left(f_{\mathrm{vol}}=100 \%\right)$. The values for each number of monomers per aggregate and for each soot volume fraction are given in Tab. 2. Consistent with Fig. 5 the values in Tab. 2 indicate an increased absorption with increased amount of coating material.

\begin{tabular}{ccccc}
$f_{\text {vol }}(\%)$ & $N=8$ & $N=64$ & $N=216$ & $N=512$ \\
\hline 75 & 1.04 & 1.04 & 1.03 & 1.03 \\
50 & 1.13 & 1.12 & 1.08 & 1.05 \\
25 & 1.24 & 1.33 & 1.26 & 1.19 \\
10 & 1.53 & 1.74 & 1.62 & 1.5
\end{tabular}

Table 2: Ratio of $C_{\mathrm{abs}}\left(f_{\mathrm{vol}}<100 \%\right)$ to $C_{\mathrm{abs}}\left(f_{\mathrm{vol}}=100 \%\right)$ at $\lambda=0.532 \mu \mathrm{m}$

Figure 6 shows the calculated depolarisation ratios for different soot volume 375 fractions at $\lambda=0.532 \mu \mathrm{m}$ (left column) and $\lambda=0.355 \mu \mathrm{m}$ (right column). The solid lines in the figure show the arithmetic mean over the ensemble of five geometries, while the shaded regions show the maximum variation within 
the ensemble. The results obtained for the slow-transition model are shown in blue, those obtained for the rapid-transition model are shown in red. The depolarisation ratio is considerably more sensitive to particle morphology than the optical cross sections. This becomes apparent when we compare Figs. 5 and 6. In Fig. 5 the shaded regions cannot be discerned. By contrast, the linear depolarisation ratios in Fig. 6 vary significantly within the ensemble of different geometries.

Figure 6 shows for $\lambda=0.532 \mu \mathrm{m}$ (left column) that the differences between the two coating models are relatively small for high soot volume fractions. For volume fractions larger than $f_{\mathrm{vol}}=25 \%$ (rows 1-4) the differences in the mean values are smaller than the range of uncertainty. However for small soot volume fractions (i.e. $f_{\mathrm{vol}}=10 \%$, bottom panel) there are significant differences in the calculated depolarisation ratios; the difference in the mean values exceeds the range of geometry-related uncertainty.

Another presentation of linear depolarisation ratios is given in Fig. 7, in which $\delta_{l}$ is plotted as a function of the soot volume fraction. The rows pertain to different numbers of monomers, while the columns display the results for the two wavelengths used as in Fig. 6. The colours are analogous to Fig,6. For all numbers of monomers the depolarisation ratios calculated with both models are in good agreement for larger soot volume fractions. For small volume fractions (i.e. $f_{\mathrm{vol}}=10 \%$ ) the resulting depolarisation ratios clearly differ. The low values of $\delta_{l}<0.25 \%$ for $N=8$ and $N=64$ in case of $\lambda=0.532 \mu \mathrm{m}$ at ${ }_{400} f_{\mathrm{vol}}=10 \%$ can be attributed to the (nearly) spherical particle shape (see Fig. 3 f for comparison).

The arithmetic mean depolarisation ratios obtained with the rapid-transition model range from $0.05 \%$ to $6.2 \%$. The corresponding values obtained with the slow-transition model range from $0.3 \%$ to $35.0 \%$. The small values at the lower end of the range obtained by the rapid-transition model correspond to small aggregates $(N=8, N=64)$ with low soot volume fraction $\left(f_{\mathrm{vol}}=10 \%\right)$ that are completely encapsulated in a spherical coating. The slow-transition coating model does not produce spherical particles for the range of soot fractions 
covered, which explains the high depolarisation ratios at the upper end of the range of computed values.

\subsection{Ultraviolet light}

To quantify the enhancement in absorption due to coating at $\lambda=0.355 \mu \mathrm{m}$ the ratio of $C_{\mathrm{abs}}\left(f_{\mathrm{vol}}<100 \%\right)$ to $C_{\mathrm{abs}}\left(f_{\mathrm{vol}}=100 \%\right)$ was calculated. The results are shown in Tab. 3. The ratios of $C_{\mathrm{abs}}\left(f_{\mathrm{vol}}<100 \%\right)$ to $C_{\mathrm{abs}}\left(f_{\mathrm{vol}}=100 \%\right)$ differ between $\lambda=0.532 \mu \mathrm{m}$ and $\lambda=0.355 \mu \mathrm{m}$. For soot volume fractions of $f_{\text {vol }}=75 \%$ the ratios do not differ much between the wavelengths. In case of soot volume fractions with $f_{\mathrm{vol}}<75 \%$ the ratios at $\lambda=0.355 \mu \mathrm{m}$ are higher for aggregates with $N=8$ than the ratios at $\lambda=0.532 \mu \mathrm{m}$. For the larger particles the ratio is lower at $\lambda=0.355 \mu \mathrm{m}$. In case of aggregates with $N=512$ and $f_{\mathrm{vol}}=50 \%$ the absorption cross section $C_{\mathrm{abs}}$ is lower than the absorption cross section for the bare aggregate. For $N=512$ and $f_{\mathrm{vol}}=25 \%$ the absorption cross section is only a little larger than for the bare aggregate. Except for one case there is an absorption enhancement at $\lambda=0.355 \mu \mathrm{m}$ which is for aggregates consisting of aggregates with $N \geq 64$ monomers lower than the enhancement at ${ }_{425} \lambda=0.532 \mu \mathrm{m}$.

\begin{tabular}{ccccc}
$f_{\text {vol }}(\%)$ & $N=8$ & $N=64$ & $N=216$ & $N=512$ \\
\hline 75 & 1.05 & 1.04 & 1.02 & 1.03 \\
50 & 1.13 & 1.09 & 1.04 & 0.99 \\
25 & 1.31 & 1.23 & 1.1 & 1.01 \\
10 & 1.71 & 1.56 & 1.36 & 1.19
\end{tabular}

Table 3: Ratio of $C_{\mathrm{abs}}\left(f_{\mathrm{vol}}<100 \%\right)$ to $C_{\mathrm{abs}}\left(f_{\mathrm{vol}}=100 \%\right)$ at $\lambda=0.355 \mu \mathrm{m}$

The general trends we observe in the ultraviolet are analogous (see Figs. 6 and 7, right column) to those we obtained for visible light in Fig. 6 (left column). For higher soot volume fractions both particle models predict rather similar depolarisation ratios, while for a volume fraction of $10 \%$ the two models differ substantially. The slow-transition model yields mean linear depolarisation 
ratios ranging from $1.4 \%$ to $52.5 \%$, while the rapid-transition model yields corresponding values from $0.2 \%$ to $17.5 \%$.

The resulting linear depolarisation ratios for coated soot particles display a strong dependency on the wavelength. Comparison of equal sizes and volume fractions in Figs. 6 and 7 reveals that $\delta_{l}$ is generally higher at $355 \mathrm{~nm}$ than at $532 \mathrm{~nm}$.

\subsection{Estimates of relative uncertainty}

The effect of a variation in geometry among aggregates with prescribed fractal parameters has been presented and discussed in the previous section. Here we consider the effect of the other sources of uncertainty listed in Sect. 2.2

\subsubsection{Effect of changes in fractal prefactor}

The fractal prefactor describes how densely the monomers are packed within each branch of the aggregate. Fig. 8 shows the depolarisation ratios obtained with the rapid transition model for two different fractal prefactors. Depolarisation ratios for aggregates with $k_{0}=0.25$ are shown in red, aggregates with $k_{0}=1.6$ in blue. The lines, shadings, panels and columns are as in Fig. 6 . For bare aggregates $\left(f_{v o l}=100 \%\right)$ and thinly coated aggregates $\left(f_{v o l}=75 \%\right)$ consisting of at least 64 monomers the effect of changes in the fractal prefactor is smaller than the uncertainties due to different geometric realisations. The uncertainty caused by changes in the fractal prefactor increases with decreasing soot volume fraction and for aggregates with $f_{v o l} \leq 25 \%$ exceeds the uncertainty due different geometric realisations. For certain configurations of number of monomers, wavelength and soot volume fraction however, the uncertainties due to changes in fractal prefactor are outweighed by the uncertainties due to different stochastic realisations of the aggregate (e.g. for $N=216, f_{v o l}=10 \%$ and $\lambda=0.355 \mu \mathrm{m})$.

The impact of changes in the fractal prefactor for the two models is illustrated in Fig. 9. Blue lines indicate the arithmetic mean of depolarisation ratios from five different stochastic realisations using the slow transition model. Red 

geometry for each case was considered, rather than computing a mean over five 
realisations 3

As for the results of the reference calculations (see subsections 3.1 and 3.2 . the differences for the different coating models and the changes in fractal dimension are most pronounced for $f_{\mathrm{vol}}=10 \%$ For the rapid-transition model increasing the fractal dimension and thus the compactness of the aggregate results in a lower depolarisation ratio, as assumed in our hypothesis. For the slow-transition model the reverse holds: With growing fractal dimension the depolarisation ratio increases. This behaviour can be seen at both wavelengths. In case of $\lambda=355 \mathrm{~nm}$ for the aggregate with $N=216$ monomers and $D_{f}=2.4$ $\left(D_{f, r e f}-0.2\right)$ the depolarisation ratio calculated with the rapid-transition model is actually higher then that calculated with the slow-transition model. However, apart from this isolated case the rapid-transition model yields lower values of the depolarisation ratio than the slow transition model. For $\lambda=355 \mathrm{~nm}$ and $f_{\mathrm{vol}}=10 \%$ the calculated depolarisation ratios using the slow-transition model are unusually high in comparison to typical field observations. Especially for $\lambda=532 \mathrm{~nm}$ the rapid-transition model reduces the uncertainty for $f_{\mathrm{vol}}=10 \%$ caused by changes in fractal dimension.

\subsubsection{Effect of changes in monomer radius}

As indicated in section 2.2 monomer radii ranging from $a=10 \mathrm{~nm}$ [1] to $a=28 \mathrm{~nm}[8]$ were reported. Two possible implications for a changed monomer radius were considered: a) changing the monomer radius and keeping the number of monomers constant thus changing the particle size and b) adjusting the number of monomers to keep the particle size fixed while changing the monomer radius.

Keeping the number of monomers constant results in an increasing volumeequivalent radius (i.e. increasing aggregate size) with increasing monomer ra-

\footnotetext{
${ }^{3}$ This does carry a risk of observing statistical artefacts. For instance, the higher depolarisation ratio for $D_{f}=2.8\left(D_{f, r e f}+0.2\right)$ compared to $D_{f}=2.6\left(D_{f, r e f}\right)$ for $f_{\mathrm{vol}}=10 \%$ and $\lambda=532 \mathrm{~nm}$ is likely to be caused by the comparison using only a single aggregate.
} 
dius. Fig. 11 shows the linear depolarisation ratios for $a=15 \mathrm{~nm}$ (red), $a=25 \mathrm{~nm}$ (green) and $a=30 \mathrm{~nm}$ (blue) and the panels are as in Fig. 6 For the same soot volume fraction and the same number of monomers the depolarisation ratio generally increases with increasing monomer radius. At both wavelengths the depolarisation ratios for $a=25 \mathrm{~nm}$ and $a=30 \mathrm{~nm}$ for a soot volume fraction $f_{v o l}=10 \%$ stronlgy overlap. The depolarisation ratios for a monomer radius of $a=10 \mathrm{~nm}$ is at both wavelengths below the values obtained by lidar field measurements as indicated by the gray dash-dotted lines. However, this does not invalidate the results, as the range of field observations reported in literature is based on relatively few measurements (see section 4).

In the other approach the volume-equivalent radius was assumed to be constant, and with changing monomer radius the number of monomers per aggregate had to be adjusted according to the relationship given in Eq. 6. Additional calculations for $a=15 \mathrm{~nm}$ and $a=30 \mathrm{~nm}$ were performed. The corresponding number of monomers per aggregate is given in Tab. 4. To save computational resources only one realisation for each aggregate was considered.

\begin{tabular}{cccc}
$r_{\text {eff,agg }}(\mathrm{nm})$ & $a=15 \mathrm{~nm}$ & $a=25 \mathrm{~nm}$ & $a=30 \mathrm{~nm}$ \\
\hline 50 & 37 & 8 & 5 \\
100 & 296 & 64 & 37 \\
150 & 1000 & 216 & 125 \\
200 & 2370 & 512 & 296
\end{tabular}

Table 4: Number of monomers $N$ corresponding to volume-equivalent radii of $50 \mathrm{~nm}, 100 \mathrm{~nm}$, $150 \mathrm{~nm}$ and $200 \mathrm{~nm}$ respectively for different monomer radii $a$. If necessary the values of $N$ calculated by using Eq. 6 were rounded to the nearest integer.

Figure 12 shows the results of the additional calculations. The linear depolarisation ratios for monomer radii of $a=15 \mathrm{~nm}$ (red), $a=25 \mathrm{~nm}$ (green) and $a=30 \mathrm{~nm}$ (blue) are shown as a function of volume-equivalent radius of the aggregate. For bare aggregates (and $f_{v o l}=25 \%$ at $355 \mathrm{~nm}$ ) the depolarisation ratio appears to be increasing slowly with increasing monomer radius. In most 

depolarisation ratios for $a=30 \mathrm{~nm}$ tend to be higher than those for $a=15 \mathrm{~nm}$, with exception of the aggregates with $r_{e f f, a g g}=200 \mathrm{~nm}$ at $532 \mathrm{~nm}$.

\subsubsection{Effect of uncertainties in the refractive index}

The depolarisation ratios computed for different refractive indices of soot and 13. The panels and particle-size ranges are similar to Fig. 6. The computations have been performed using only one aggregate realisation. The left column of the figure shows soot- $1\left(m_{\text {soot, } 1}=1.76+i 0.63\right)$ with organic $\left(m_{\text {organic }}=\right.$ $1.53+i 0.0055)\left(\right.$ dark red) and sulphate coating $\left(m_{\mathrm{SO}_{4}}=1.43+i 10^{-8}\right)$ (blue), and soot- $2\left(m_{\text {soot }, 2}=1.95+i 0.79\right)$ with organic (orange) and sulphate coating (green).

A change in the refractive index of the coating material has the largest effect for low soot volume fractions. By contrast, a change in the refractive index of soot can impact the depolarsation ratio both at low and at high volume fractions. In general, changes in the refractive index have a stronger effect on the depolarisation ratio than the differences we observe when varying the geometry of the aggregates (compare to the shaded regions in Fig. 6).

The depolarisation ratios for different refractive indices at $\lambda=0.355 \mu \mathrm{m}$ (see right column of Fig. 13 have to be compared with caution to the values for ${ }_{555} \lambda=0.532 \mu \mathrm{m}$ as the chosen refractive indices do not refer to the same type of soot and mildly absorbing coating, respectively, as pointed out in section 2.2 . The right column of Fig. 13 shows soot-3 $\left(m_{\text {soot, } 3}=1.66284+i 0.71528\right)$ with toluene $\left(m_{\text {toluene }}=1.632+i 0.047\right)(\mathrm{red})$ and sulphate coating $\left(m_{\mathrm{SO}_{4}}=\right.$ $\left.1.45+i 10^{-8}\right)($ blue $)$, and soot-4 $\left(m_{\text {soot }, 4}=1.68586+i 0.67251\right)$ with toluene (orange) and sulphate coating (green). The relatively small differences between the two different soot types, which is in every considered case smaller than the difference between the coating types, is most likely due to the relatively small change in refractive index. For soot volume fractions of $f_{\mathrm{vol}}=75 \%, f_{\mathrm{vol}}=50 \%$ and $f_{\text {vol }}=25 \%$ the aggregates coated with weakly absorbing toluene have a 

soot volume fractions (rows) and wavelengths (columns) calculated using the rapid transition model in conjunction with different overlap factors. The results for aggregates with point-contacting monomers $\left(C_{o v}=0.0\right)$ are shown in red, 

ping increases $\delta_{l}$, the reverse happens for all aggregates for $f_{\text {vol }}=10 \%$, as well as for aggregates with $N=8$ and $f_{\text {vol }} \leq 50 \%$.

\subsubsection{Summary of relative uncertainties}

We calculated the (maximum) relative uncertainty ${ }^{4}$ for each aggregate size and soot volume fraction. It was found that changes in the soot volume fraction had a larger impact on the relative uncertainty than the particle size. For each volume fraction, we take the maximum relative uncertainty over all particle sizes (not considering the spherically coated aggregates for $N=8$ with $\left.f_{\mathrm{vol}}=25 \%, f_{\mathrm{vol}}=10 \%\right)$. The different relative uncertainties $\Delta \delta_{l}$ for $\lambda=532 \mathrm{~nm}$ are shown in Tab. 5. The subscripts refer to the different sources of uncertainty, namely, aggregate geometry (geo), fractal prefactor $\left(k_{0}\right)$, fractal dimension $\left(D_{f}\right)$, monomer radius (rad), overlapping monomers (ov), refractive index of aggregate (agg) and coating (coat). In case of the uncertainty due to changes in monomer radius only the effects of aggregates with constant volume-equivalent aggregate

\footnotetext{
${ }^{4}$ The relative uncertainty is defined as $\Delta \delta_{1}=100 \% \times\left|\delta_{1, \text { ref }}-\delta_{1, \mathrm{x}}\right| / \delta_{1, \text { ref }}$, where $\delta_{1, \text { ref }}$ denotes a reference or mean value, and $\delta_{1, \mathrm{x}}$ represents the maximum deviation from the reference.
} 
radius were considered. The total relative uncertainty was calculated by assuming that the different sources of error are statistically independent of each other, so that

$$
\Delta_{\text {tot }} \delta_{l}=\sqrt{\Delta_{\text {geo }} \delta_{l}^{2}+\Delta_{\mathrm{k}_{0}} \delta_{l}^{2}+\Delta_{\mathrm{D}_{\mathrm{f}}} \delta_{l}^{2}+\Delta_{\mathrm{rad}} \delta_{l}^{2}+\Delta_{\mathrm{ov}} \delta_{l}^{2}+\Delta_{\mathrm{agg}} \delta_{l}^{2}+\Delta_{\text {coat }} \delta_{l}^{2}} .
$$
uncertainty can be seen as a lower bound of the overall uncertainty.

\begin{tabular}{ccccccccc}
$f_{\mathrm{vol}}(\%)$ & $\Delta_{\text {geo }} \delta_{l}$ & $\Delta_{\mathrm{k}_{0}} \delta_{l}$ & $\Delta_{\mathrm{D}_{\mathrm{f}}} \delta_{l}$ & $\Delta_{\mathrm{rad}} \delta_{l}$ & $\Delta_{\mathrm{ov}} \delta_{l}$ & $\Delta_{\mathrm{agg}} \delta_{l}$ & $\Delta_{\text {coat }} \delta_{l}$ & $\Delta_{\text {tot }} \delta_{l}$ \\
\hline 100 & 25.2 & 79.6 & 32.5 & 54.5 & 41.0 & 20.4 & - & 114.4 \\
75 & 27.4 & 74.1 & 39.5 & 61.6 & 40.1 & 18.8 & 6.1 & 116.6 \\
50 & 44.2 & 80.2 & 32.8 & 62.6 & 30.1 & 18.3 & 10.5 & 121.4 \\
25 & 49.6 & 59.1 & 59.7 & 33.7 & 32.0 & 10.9 & 11.9 & 109.3 \\
10 & 41.0 & 93.5 & 204.0 & 46.7 & 21.3 & 32.5 & 56.2 & 242.7
\end{tabular}

Table 5: Maximum relative uncertainties and total relative uncertainty in $\%$ for different $f_{\text {vol }}$ at $\lambda=532 \mathrm{~nm}$

In case of the fractal prefactor there is additionally a dependence of uncertainty on the number of monomers, as can be inferred from Fig. 8 . To address the size dependence of the uncertainty due to changes in fractal prefactors we 


\begin{tabular}{ccc}
$f_{\text {vol }}(\%)$ & $\Delta_{\mathrm{k}_{0}, \text { small }} \delta_{l}(\%)$ & $\Delta_{\mathrm{k}_{0}, \text { large }} \delta_{l}$ \\
\hline 100 & 79.6 & 4.9 \\
75 & 74.1 & 14.5 \\
50 & 80.2 & 14.0 \\
25 & 59.1 & 38.5 \\
10 & 93.2 & 18.4
\end{tabular}

Table 6: Uncertainty due to changes in fractal prefactor for different soot volume fraction $f_{\mathrm{vol}}$ at $\lambda=0.532 \mu \mathrm{m}$ compared for "small" aggregates $(N=8, N=64)$ and "large" aggregates $(N=216, N=512)$

decided to refer to aggregate consisting either of $N=8$ or $N=64$ monomers as "small" and to aggregate consisting of $N=216$ and $N=512$ monomers as "large". The corresponding maximum uncertainties at $\lambda=0.532 \mu \mathrm{m}$ for both small and large aggregates for each of the considered soot volume fraction are given in Tab. 6. As can be seen the uncertainty is substantially lower for large aggregates. For the fractal dimension the dependence is rather ambiguous (see Tab. 7p: For $f_{v o l}=100 \%$ and $f_{25 \%}$ the uncertainty decreases for the large aggregates. For $f_{v o l}=75 \%$ and $f_{50 \%}$ the calculated uncertainties are higher for the large aggregates, but the increase is smaller than the decrease in the other two cases. However, in case of $f_{v o l}=10 \%$ the highest uncertainties due to changes in the fractal dimensions are for larger aggregates. If knowledge about the aggregate size exists and in case of the fractal dimension additional knowledge of the coating thickness, the total uncertainty due to changes in fractal prefactor can be reduced. A likely explanation for the high sensitivity of $\delta_{1}$ to changes in fractal parameters for small aggregates is that for the latter the fractal structure, hence the fractal parameters, are poorly defined, due to the small number of monomers. Thus the observed sensitivity of $\delta_{1}$ to changes in the fractal parameters may simply be a statistical artifact.

The uncertainties at $\lambda=355 \mathrm{~nm}$ are shown in Tab. 8. In general, the relative uncertainties $\Delta_{\text {geo }} \delta_{l}$ are smaller at $355 \mathrm{~nm}$ than at $532 \mathrm{~nm}$, except for 


\begin{tabular}{ccc}
$f_{\text {vol }}(\%)$ & $\Delta_{\mathrm{D}_{\mathrm{f}}, \text { small }} \delta_{l}(\%)$ & $\Delta_{\mathrm{D}_{\mathrm{f}}, \text { large }} \delta_{l}$ \\
\hline 100 & 32.5 & 14.4 \\
75 & 29.7 & 39.5 \\
50 & 27.4 & 31.4 \\
25 & 59.7 & 29.3 \\
10 & 101.3 & 204.0
\end{tabular}

Table 7: Uncertainty due to changes in fractal dimension for different soot volume fraction $f_{\text {vol }}$ at $\lambda=0.532 \mu \mathrm{m}$ compared for "small" aggregates $(N=8, N=64)$ and "large" aggregates $(N=216, N=512)$

$f_{\mathrm{vol}}=75 \%$ at which the relative uncertainties are similar. At both wavelengths the maximum values of the geometry-related relative uncertainty have their peak values at $f_{\mathrm{vol}}=50 \%$ and $f_{\mathrm{vol}}=25 \%$.

\begin{tabular}{ccccccccc}
$f_{\mathrm{vol}}(\%)$ & $\Delta_{g e o} \delta_{l}$ & $\Delta_{\mathrm{k}_{0}} \delta_{l}$ & $\Delta_{\mathrm{D}_{\mathrm{f}}} \delta_{l}$ & $\Delta_{\mathrm{rad}} \delta_{l}$ & $\Delta_{\mathrm{ov}} \delta_{l}$ & $\Delta_{\mathrm{agg}} \delta_{l}$ & $\Delta_{\text {coat }} \delta_{l}$ & $\Delta_{\mathrm{tot}} \delta_{l}$ \\
\hline 100 & 18.8 & 75.1 & 37.5 & 57.3 & 44.8 & 4.3 & - & 112.7 \\
75 & 26.9 & 72.2 & 31.2 & 54.9 & 41.8 & 2.2 & 9.0 & 109.1 \\
50 & 37.7 & 80.8 & 51.1 & 39.4 & 30.6 & 2.6 & 14.6 & 115.2 \\
25 & 32.3 & 44.2 & 45.6 & 24.4 & 44.8 & 4.5 & 23.8 & 90.9 \\
10 & 24.1 & 40.4 & 165.4 & 27.6 & 25.2 & 23.7 & 90.9 & 199.5
\end{tabular}

Table 8: Maximum relative uncertainties and total relative uncertainty in $\%$ for different $f_{\text {vol }}$ for $\lambda=355 \mathrm{~nm}$

As for $\lambda=0.532 \mu \mathrm{m}$ we considered the uncertainty due to changes in fractal parameters for small $(N=8, N=64)$ and large aggregates $(N=216, N=512)$. The corresponding values are given for the fractal prefactor in Tab. 9 and for the fractal dimension in Tab. 10. As again can be seen in Fig. 8 the uncertainty due to changes in fractal prefactor depends on the size. With the exception of $f_{\text {vol }}=25 \%$ the uncertainty is lower for the large aggregates. In case of the 655 fractal dimension the size dependence of the uncertainty is less ambiguous as at $\lambda=0.532 \mu \mathrm{m}$, as with exception of $f_{v o l}=10 \%$ the uncertainty for the large 


\begin{tabular}{ccc}
$f_{\text {vol }}(\%)$ & $\Delta_{\mathrm{k}_{0}, \text { small }} \delta_{l}(\%)$ & $\Delta_{\mathrm{k}_{0}, \text { large }} \delta_{l}$ \\
\hline 100 & 75.1 & 3.5 \\
75 & 72.2 & 2.6 \\
50 & 80.8 & 13.9 \\
25 & 16.2 & 44.2 \\
10 & 40.4 & 19.7
\end{tabular}

Table 9: Uncertainty due to changes in fractal prefactor for different soot volume fraction $f_{\mathrm{vol}}$ at $\lambda=0.355 \mu \mathrm{m}$ compared for "small" aggregates $(N=8, N=64)$ and "large" aggregates $(N=216, N=512)$

\begin{tabular}{ccc}
$f_{\text {vol }}(\%)$ & $\Delta_{\mathrm{D}_{\mathrm{f}}, \text { small }} \delta_{l}(\%)$ & $\Delta_{\mathrm{D}_{\mathrm{f}}, \text { large }} \delta_{l}$ \\
\hline 100 & 37.5 & 21.9 \\
75 & 31.2 & 26.9 \\
50 & 51.1 & 25.0 \\
25 & 45.6 & 18.6 \\
10 & 58.7 & 165.4
\end{tabular}

Table 10: Uncertainty due to changes in fractal dimension for different soot volume fraction $f_{\mathrm{vol}}$ at $\lambda=0.355 \mu \mathrm{m}$ compared for "small" aggregates $(N=8, N=64)$ and "large" aggregates $(N=216, N=512)$

aggregates is smaller than the uncertainty for the small aggregates.

\section{Discussion}

Field measurements of the linear backscattering depolarisation ratio at $\lambda=532 \mathrm{~nm}$ ported values in the range of 1.5 to $11 \%$. In our calculations based on the rapid-transition model we obtained, depending on the soot volume fraction and number of monomers, mean linear depolarisation ratios in the range of 0.055 $6.2 \%$ for the reference calculations for aggregates consisting of point-contacting 665 


\begin{tabular}{llll}
$\delta_{l}(\%)$ & location & type & reference \\
\hline $6-11$ & Lindenberg, Germany & aged BBA & {$[55]$} \\
$5-8$ & Tokyo, Japan & aged smoke & {$[56]$} \\
$1.5-3$ & Leipzig, Germany & smoke & {$[57]$} \\
$<3$ & Fairbanks, USA & fresh smoke & {$[58]$} \\
5 & Fairbanks, USA & aged smoke & {$[58]$} \\
$15-17$ & Praia, Cape Verde & BBA & {$[34]$} \\
$15-23$ & Praia, Cape Verde & probably BBA & {$[34]$} \\
$3-7$ & Praia, Cape Verde & smoke & {$[59]$} \\
$<2-5$ & North America (various flight campaigns) & fresh smoke & {$[60]$} \\
$3-8$ & North America (various flight campaigns) & aged smoke & {$[60]$} \\
$<3$ & Manaus, Brazil & aged smoke & {$[35]$} \\
$6-8$ & North-East Germany & aged smoke & {$[61]$} \\
$5.8-7.8$ & US East Coast & smoke & {$[36$} \\
$7.8-10.8$ & Denver, USA & smoke & {$[36]$} \\
$<5$ & Western Mediterranean Sea & BBA & {$[38$}
\end{tabular}

Table 11: $\delta_{l}$ at $532 \mathrm{~nm}$ obtained from various field campaigns. The given type always refers the the classification given in the cited reference. We assume that smoke and biomass burning aerosol (BBA) refer to (coated) soot aerosol particles. 


\begin{tabular}{llll}
$f_{\text {vol }}(\%)$ & age & location & reference \\
\hline $7-24$ (median: 15$)$ & $<12 h$ & Mexico City, Mexico & {$[5]$} \\
6,13 & (not reported) & Lindenberg, Germany & {$[6]$} \\
$7 \pm 8$ & $<1$ day & Mexico City, Mexico & {$[7]$} \\
(not quantitatively reported) & $\sim 1-2 h$ & Los Alamos, USA & {$[8]$}
\end{tabular}

Table 12: Examples of soot volume fractions $f_{\mathrm{vol}}$ of sampled atmospheric soot particles reported in the literature

Aged soot particles in the atmosphere often have low soot volume fractions [5, 6, 7, 8, Some reported values of soot volume fraction are shown in Tab. 12 In [8] 1026 soot particles from forest fire smoke were analysed, while the soot volume fraction was not quantitatively reported, it is worth noting, that $50 \%$ of the particles were described as being heavily coated, $34 \%$ as partly coated and $4 \%$ as uncoated or very thinly coated. The remaining $12 \%$ were described as soot partly embedded in coating material. This particle count might be biased low, as very thick coating might lead to the soot monomers being indistinguishable and therefore this particles might be wrongly classified as not soot containing 675 [8. The reported soot particle age in [5, 8] indicates fast coating processes in the atmosphere. The range of reported soot volume fractions for atmospheric soot particles $\left(f_{\mathrm{vol}}<24 \%\right)$, as given in Tab. 12 indicate that our simulation results for soot volume fractions of $f_{\mathrm{vol}}=10 \%$ and $f_{\mathrm{vol}}=25 \%$ (see rows 4 and 5 in Figs. 6, 15 are most relevant for comparing or modelling results to lidar field measurements. The higher soot volume fractions, on the other hand, are more relevant for comparison with laboratory measurements than with field measurements.

As a reference case we assumed aggregates consisting of monomers with a radius of $a=25 \mathrm{~nm}$. For $f_{\mathrm{vol}}=10 \%$ this corresponds to an aggregate with a vol685 ume equivalent radius of $a_{\mathrm{eff}}=320 \mathrm{~nm}(N=216)$ and $a_{\mathrm{eff}}=430 \mathrm{~nm}(N=512)$ respectively (see Eqs. 6, 7). Reported median and mean volume equivalent radii of $a_{\text {eff,median }}=145 \mathrm{~nm} \mathrm{[5]} \mathrm{and} a_{\text {eff,mean }}=206 \mathrm{~nm}$ [7] suggest that most 
atmospheric soot aerosol particles are smaller then the largest particles modelled in this study. However scattering by particles increases with particle size. Therefore relatively few large particles can still have a considerable impact on bulk scattering properties 62 .

According to table 5 the uncertainty in our model estimates for such particles is close to $\Delta_{t o t} \delta_{l} \approx 245 \%(\lambda=0.532 \mu \mathrm{m})$ and $\Delta_{t o t} \delta_{l} \approx 200 \%(\lambda=0.355 \mu \mathrm{m})$. For certain configurations of input parameters the modell may still give results not consistent with lidar field measurements.

By contrast, the slow-transition model yields depolarisation ratios of $\delta_{l}=$ $35.0 \%$ for $f_{\mathrm{vol}}=10 \%$ and $N=512$ assuming point-contacting monomers and sulphate coating, which is higher than the depolarisation ratio of $\delta_{l}=5.7 \%$ resulting from the rapid-transition model. These values obtained with the slow transition model lie outside the range of observed field measurements. Also, the slow-transition model used here yields results for $\delta_{l}$ that are higher than values of $\delta_{l}=16 \%$ as reported in [26]. The main difference is that the slowtransition model in the present study does account for the compaction of the aggregate with decreasing soot volume fraction, while the model in [26] does not. Thus an increase of the compactness of the aggregate with decreasing soot volume fraction without changing the critical coating diameter for the onset of sphericity results in an increase of the depolarisation ratio. By contrast, in the rapid-transition aggregate model the combined effect of reducing the critical coating diameter and of increasing the aggregate's compactness resulted in depolarisation ratios consistent with the existing lidar field measurements.

To check the consistency of the calculated depolarisation ratios at $355 \mathrm{~nm}$ they were gauged against results of lidar field measurements reported in the literature, which we summarise in Tab. 13 .

These field measurements report depolarisation ratios at $355 \mathrm{~nm}$ ranging ${ }_{715}$ from 2-25\%. Most notable are the relatively large values reported in [20, 34, 36, which pertain to non-dust containing aerosols. The calculations for an aggregate of point-contacting monomers with sulphate coating using the rapid-transition model cover a range of $\delta_{l}$ from 1.7 to $20.3 \%$, which largely lies within the range 


\begin{tabular}{llll}
$\delta_{l}(\%)$ & location & type & reference \\
\hline $4-5$ & Manaus, Brazil & aged biomass burning & {$[33$} \\
$15-19$ & Praia, Cape Verde & BBA & {$[34]$} \\
$21-25$ & Praia, Cape Verde & probably BBA & {$[34]$} \\
$<3$ & Manaus, Brazil & aged smoke & {$[35]$} \\
$7-13$ & Leipzig, Germany & aged biomass burning & {$[20]$} \\
$16-24$ & Denver, USA & pure smoke & {$[36]$} \\
$2-6$ & Kazan, Russia & pure BBA & {$[37]$} \\
$2-5$ & Elandsfontein, South Africa & BBA & {$[39$}
\end{tabular}

Table 13: $\delta_{l}$ at $355 \mathrm{~nm}$ obtained from various field campaigns. The given type always refers the the classification given in the cited reference. We assume that smoke and biomass burning aerosol (BBA) refer to (coated) soot aerosol particles.

of reported values obtained from lidar field measurements. The slow-transition model yields values of $\delta_{l}$ up to $55 \%$ for $f_{\mathrm{vol}}=10 \%$; this is clearly inconsistent with the values obtained in lidar field measurements.

The values calculated for the linear depolarisation ratio at $355 \mathrm{~nm}$ for $f_{\mathrm{vol}}=$ $10 \%$ have the same order of magnitude $(\sim 20 \%)$ as results presented in 63 . Those values were obtained in order to reproduce the measurements by [36] using different particle models, all having relatively small $f_{\text {vol }}$, namely a closed cell model with $0.4 \%<f_{\text {vol }}<0.5 \%$, a model of two contacting spheres each encapsulating an aggregate with $2.0 \%<f_{\mathrm{vol}}<8.0 \%$ and concentric core-mantle spheroids for different combinations of axis ratio and soot volume fraction with $2.0 \%<f_{\mathrm{vol}}<12.5 \%$.

The depolarisation ratios obtained with the rapid-transition model are more consistent with the reported field measurements than those obtained with either the slow-transition model or the model used in [26]. This is remarkable, since the morphological differences among these three models are rather subtle. These results illustrate the high sensitivity of the depolarisation ratio to the particles' 735 geometry. 
However, comparisons of model results with field observations provide us with little more than a consistency check; they cannot be interpreted as reliable quantitative evidence. This is due to a number of unknowns in the experimental data. The field measurements do not provide us with information on the aggregates' geometry (e.g. fractal parameters) and the soot volume fraction. Nor is it always trivial to determine whether or not the observed plumes were composed of pure soot aerosols or of mixtures contaminated with other compounds, such as dust.

Our main hypothesis was that the depolarisation ratio of the model particles can be controlled by the the mode of transition from film-coating to spherical coating. As more coating material is added to the soot aggregate, a faster transition to spherically coated aggregates was expected to result in lower depolarisation ratios as compared to a slow-transition model. This hypothesis was largely confirmed by our results, although with some rather interesting reserva750 tions.

It was hypothesised that a fast transition to spherical coating can be achieved by (i) choosing a relatively small value of the critical radius that marks to onset of spherical growth; and (ii) allowing the aggregate to become more compact as more coating material is added. The choice of the critical radius had, indeed, a profound impact on the depolarisation ratio, as hypothesised. However, an increase in fractal dimension may give rise to two competing effects.

- A more compact aggregate is more readily encapsulated by a spherical shell with no parts of the aggregate sticking out of the shell. This is expected to result in a low depolarisation ratio, especially for heavily coated aggregates.

- A more compact aggregate would give rise to more electromagnetic interaction among the monomers, so the optical properties should be less similar to independently scattering monomers than in a lacy aggregate. This may increase the depolarisation ratio, especially in thinly coated aggregates. 
The latter effect explains why the depolarisation ratios computed in [26] were lower than those computed with the slow transition model. Both models use the same critical radius that defines the onset of spherical growth. However, the model in [26] neglects compaction of the aggregates with increasing coating

\section{Summary and Conclusions}

A main goal in fundamental aerosol optics research is to understand the relation between morphological and optical properties. In particular, we want to identify those morphological features that have a dominant impact on the optical properties. This is often much easier for integral optical properties, such as the total scattering and absorption cross sections, than for differential scattering properties. It is particularly challenging for quantities, such as $\delta_{l}$, that are exceedingly sensitive to even small variations in particle morphology. The findings of this study have allowed us to make some encouraging progress in this regard. The results indicate that one of the essential morphological 

the rapid transition to a spherical coating, at least for soot volume fractions higher than $25 \%$.

Here we extended the computations in [26, which were limited to a visible wavelength of $532 \mathrm{~nm}$, to include a UV wavelength of $355 \mathrm{~nm}$. For both wave-

features of soot aerosols is the speed of transition from the nonspherical to the spherical coating regime as more coating material is added. In the present study we parameterised the onset of the spherical-coating regime by defining a critical diameter, which was based on an educated guess. However, we expect that this critical diameter should be dependent on the hygroscopicity of the soot aggregate and/or the surface tension of the coating material. Suitable refinements of our model will have to depend on more guidance from laboratory studies, such as the ones reported in 31].

In choosing the critical radius we can get some rough guidance from field measurements with lidar instruments. However, we know from earlier modelling studies (e.g. [36, 26]) that it is challenging to reproduce lidar field observations of the linear backscattering depolarisation ratio $\delta_{l}$ of soot aerosols with models. There may be a certain risk that the model in [26] overestimates $\delta_{l}$ for large, heavily coated soot particles, which may indicate that those model particles are not sufficiently spherical. This observation lead us to hypothesise that one of the essential morphological properties in soot-particles is the mode of transition from a thin film-coating to a spherical shell; we proposed to account for a relatively rapid transition from nonspherical to spherical shape as more coating material is added to the soot aggregate. This can be achieved by (i) reducing the critical diameter which defines the onset of sphericity in the coated aggregate model; and (ii) taking the compaction of soot into account as more coating material is added. Our main hypothesis was that the depolarisation ratio is highly sensitive to this speed of transition. This hypothesis is supported by our results. However, the compaction of soot can also enhance electromagnetic interaction among the monomers, which can increase the depolarisation. This phenomenon seems to somewhat diminish the depolarisation-reducing effect of lengths we found that our rapid-transition coated aggregate model produced $\delta_{l}$ 
values that were largely consistent with lidar field observations of soot plumes.

A long-term goal of this study is to develop a model particle that can be employed in retrieval algorithms and in chemical data assimilation. For such purposes it is essential to have not only a reliable aerosol optics model, but also a realistic estimate of the model uncertainties. Using the rapid-transition model, different sources of uncertainty of the model results were examined at $532 \mathrm{~nm}$ and $355 \mathrm{~nm}$. Depending on the soot volume fraction the total relative uncertainty in $\delta_{l}$ at $532 \mathrm{~nm}$ ranges between 109 and $243 \%$. At $355 \mathrm{~nm}$ the total relative uncertainty in $\delta_{l}$ ranges from 90.9 to $200 \%$. Model errors caused by a limited knowledge of the extent of overlapping between neighbouring monomers or the refractive index of both aggregate and coating material could be reduced as more reliable measurements become available. However, the uncertainty related to our lack of precise knowledge of the aggregate geometry cannot be reduced. The values for this type of uncertainty range from 25 to $50 \%$ at $532 \mathrm{~nm}$ and 19 to $38 \%$ at $355 \mathrm{~nm}$ depending on the soot volume fraction. This can be considered a lower bound for the modelling uncertainty in $\delta_{l}$.

\section{Acknowledgements}

We are grateful to Dan Mackowski for providing us with his cluster-cluster aggregation program. Maxim Yurkin and Alfons G. Hoekstra are acknowledged for making their ADDA code publicly available. F. Kanngießer and M. Kahnert acknowledge funding by the Swedish Research Council (Vetenskapsrådet; dnr 2016-03499); M. Kahnert acknowledges funding by the Swedish National Space Board (Rymdstyrelsen; dnr 100/16).

\section{References}

${ }_{850}$ [1] T. C. Bond, R. W. Bergstrom, Light absorption by carbonaceous parti1. cles: An investigative review, Aerosol Sci. Technol. 40 (1). doi:10.1080/ 02786820500421521 
[2] P. Chýlek, G. B. Lesins, G. Videen, J. G. D. Wong, R. G. Pinnick, D. Ngo, J. D. Klett, Black carbon and absorption of solar radiation by clouds, J. Geophys. Res. 101 (D18) (1996) 23365-23371. doi:10.1029/96JD01901.

[3] S. C. Anenberg, K. Talgo, S. Arunachalam, P. Dolwick, C. Jang, J. J. West, Impacts of global, regional, and sectoral black carbon emission reductions on surface air quality and human mortality, Atmos. Chem. Phys. 11 (14) (2011) 7253-7267. doi:10.5194/acp-11-7253-2011.

[4] R. Zhang, A. F. Khalizov, J. Pagels, D. Zhang, H. Xue, P. H. McMurry, Variability in morphology, hygroscopicity, and optical properties of soot aerosols during atmospheric processing, Proc Natl Acad Sci U S A 105 (30) (2008) 10291-10296. doi:10.1073/pnas.0804860105.

[5] K. Adachi, P. R. Buseck, Internally mixed soot, sulfates, and organic matter in aerosol particles from mexico city, Atmos. Chem. Phys. 8 (21) (2008) 6469-6481. doi:10.5194/acp-8-6469-2008

[6] A. Worringen, M. Ebert, T. Trautmann, S. Weinbruch, G. Helas, Optical properties of internally mixed ammonium sulfate and soot particles-a study of individual aerosol particles and ambient aerosol populations, Appl. Opt. 47 (21) (2008) 3835-3845. doi:10.1364/A0.47.003835

[7] K. Adachi, S. H. Chung, P. R. Buseck, Shapes of soot aerosol particles and implications for their effects on climate, J. Geophys. Res. 115 (D15) (2010) n/a-n/a. doi:10.1029/2009JD012868

[8] S. China, C. Mazzoleni, K. Gorkowski, A. C. Aiken, M. K. Dubey, Morphology and mixing state of individual freshly emitted wildfire carbonaceous particles, Nat. Commun. 4 (2122). doi:10.1038/ncomms3122.

[9] M. Kahnert, T. Nousiainen, H. Lindqvist, Models for integrated and differential scattering optical properties of encapsulated light absorbing carbon aggregates, Opt. Express 21 (7) (2013) 7974-7993. doi:10.1364/OE.21. 007974 
[10] M. Kahnert, T. Nousiainen, H. Lindqvist, Review: Model particles in atmospheric optics, J. Quant. Spectrosc. Radiat. Transfer 146 (Supplement C) (2014) 41 - 58, electromagnetic and Light Scattering by Nonspherical Particles XIV. doi:https://doi.org/10.1016/j.jqsrt.2014.02.014

[16] Y. Wu, T. Cheng, L. Zheng, H. Chen, H. Xu, Single scattering properties
of semi-embedded soot morphologies with intersecting and non-intersecting 905

[11] F. Liu, J. Yon, A. Bescond, On the radiative properties of soot aggregates part 2: Effects of coating, J. Quant. Spectrosc. Radiat. Transfer 172 (Supplement C) (2016) 134-145. doi:10.1016/j.jqsrt.2015.08.005.

[12] J. Dong, J. Zhao, L. Liu, Morphological effects on the radiative properties of soot aerosols in different internally mixing states with sulfate, J. Quant. Spectrosc. Radiat. Transfer 165 (Supplement C) (2015) 43-55. doi:10. $1016 / j \cdot j q s r t .2015 .06 .025$

[13] Y. Wu, T. Cheng, L. Zheng, H. Chen, Models for the optical simulations of fractal aggregated soot particles thinly coated with non-absorbing aerosols, J. Quant. Spectrosc. Radiat. Transfer 182 (Supplement C) (2016) 1-11. doi:10.1016/j.jqsrt.2016.05.011.

[14] Y. Wu, T. Cheng, X. Gu, L. Zheng, H. Chen, H. Xu, The single scattering properties of soot aggregates with concentric coreshell spherical monomers, J. Quant. Spectrosc. Radiat. Transfer 135 (Supplement C) (2014) 9 - 19. doi:https://doi.org/10.1016/j.jqsrt.2013.11.009.

[15] K. Liou, Y. Takano, P. Yang, Light absorption and scattering by aggregates: Application to black carbon and snow grains, J. Quant. Spectrosc. Radiat. Transfer 112 (10) (2011) 1581 - 1594. doi:https://doi.org/10.1016/j. jqsrt.2011.03.007. surfaces of absorbing spheres and non-absorbing host, J. Quant. Spectrosc.

q Radiat. Transfer 157 (Supplement C) (2015) 1 - 13. doi:https://doi. org $/ 10.1016 / j \cdot j q s r t .2015 .02 .006$. 
[17] A. Bescond, J. Yon, T. Girasole, C. Jouen, C. Roz, A. Coppalle, Numerical org/10.1016/j.jqsrt.2012.10.011.

[18] A. H. Omar, D. M. Winker, M. A. Vaughan, Y. Hu, C. R. Trepte, R. A.

[19] S. Groß, V. Freudenthaler, M. Wirth, B. Weinzierl, Towards an aerosol

[20] A. J. Illingworth, H. W. Barker, A. Beljaars, M. Ceccaldi, H. Chepfer, N. Clerbaux, J. Cole, J. Delano, C. Domenech, D. P. Donovan, S. Fukuda,

[22] Y. Wu, T. Cheng, L. Zheng, H. Chen, Optical properties of the semiexternal mixture composed of sulfate particle and different quantities of 
soot aggregates, J. Quant. Spectrosc. Radiat. Transfer 179 (Supplement C) (2016) 139 -148. doi:https://doi.org/10.1016/j.jqsrt.2016.03.012.

[24] R. Ghazi, J. S. Olfert, Coating mass dependence of soot aggregate re-

[23] R. P. Bambha, M. A. Dansson, P. E. Schrader, H. A. Michelsen, Effects of volatile coatings and coating removal mechanisms on the morphology of graphitic soot, Carbon 61 (2013) 80 - 96. doi:10.1016/j.carbon.2013. 04.070 structuring due to coatings of oleic acid and dioctyl sebacate, Aerosol Sci. Technol. 47 (2) (2013) 192-200. doi:10.1080/02786826.2012.741273.

[25] E. G. Schnitzler, A. Dutt, A. M. Charbonneau, J. S. Olfert, W. Jger, Soot aggregate restructuring due to coatings of secondary organic aerosol derived from aromatic precursors, Environ. Sci. Technol. 48 (24) (2014) 14309-14316, pMID: 25390075. doi:10.1021/es503699b.

[26] M. Kahnert, Optical properties of black carbon aerosols encapsulated in a shell of sulfate: comparison of the closed cell model with a coated aggregate model, Opt. Express 25 (20) (2017) 24579-24593. doi:10.1364/OE.25. 024579

[27] C. M. Sorensen, Light scattering by fractal aggregates: A review, Aerosol Sci. Technol. 35 (2001) 648-687. doi:10.1080/02786820117868.

[28] D. W. Mackowski, A simplified model to predict the effects of aggregation on the absorption properties of soot particles, J. Quant. Spectrosc. Radiat. Transfer 100 (1) (2006) 237-249, vIII Conference on Electromagnetic and Light Scattering by Nonspherical Particles. doi:10.1016/j.jqsrt.2005. 11.041

[29] K. Adachi, S. H. Chung, H. Friedrich, P. R. Buseck, Fractal parameters of individual soot particles determined using electron tomography: Implications for optical properties, J. Geophys. Res. 112 (D14) (2007) n/a-n/a. doi:10.1029/2006JD008296 
[30] M. Kahnert, T. Nousiainen, H. Lindqvist, M. Ebert, Optical properties of light absorbing carbon aggregates mixed with sulfate: assessment of different model geometries for climate forcing calculations, Opt. Express 20 (9) (2012) 10042-10058. doi:10.1364/OE.20.010042.

[31] X. Pei, M. Hallquist, A. C. Eriksson, J. H. Pagels, N. M. Donahue, T. Mentel, B. Svenningsson, W. Brune, R. K. Pathak, Morphological transformation of soot: investigation of microphysical processes during the condensation of sulfuric acid and limonene ozonolysis product vapors, Atmos. Chem. Phys. Discuss. 2017 (2017) 1-30. doi:10.5194/acp-2017-769

[32] U. Wandinger, Lidar - Range-resolved optical remote sensing of the atmosphere, Springer New York, 2005, Ch. Introduction to lidar, pp. 1-18.

[33] A. Ansmann, H. Baars, M. Tesche, D. Müller, D. Althausen, R. Engelmann, T. Pauliquevis, P. Artaxo, Dust and smoke transport from africa to south america: Lidar profiling over cape verde and the amazon rainforest, Geophys. Res. Lett. 36 (11) (2009) n/a-n/a, l11802. doi: 10.1029/2009GL037923.

[34] S. Groß, M. Tesche, V. Freudenthaler, C. Toledano, M. Wiegner, A. Ansmann, D. Althausen, M. Seefeldner, Characterization of saharan dust, marine aerosols and mixtures of biomass-burning aerosols and dust by means of multi-wavelength depolarization and raman lidar measurements during samum 2, Tellus B: Chemical and Physical Meteorology 63 (4) (2011) 706724. doi:10.1111/j.1600-0889.2011.00556.x.

[35] H. Baars, A. Ansmann, D. Althausen, R. Engelmann, B. Heese, D. Müller, P. Artaxo, M. Paixao, T. Pauliquevis, R. Souza, Aerosol profiling with lidar in the amazon basin during the wet and dry season, J. Geophys. Res. 117 (D21) (2012) n/a-n/a. doi:10.1029/2012JD018338.

[36] S. P. Burton, J. W. Hair, M. Kahnert, R. A. Ferrare, C. A. Hostetler, A. L. Cook, D. B. Harper, T. A. Berkoff, S. T. Seaman, J. E. Collins, 
M. A. Fenn, R. R. Rogers, Observations of the spectral dependence of linear particle depolarization ratio of aerosols using nasa langley airborne high spectral resolution lidar, Atmos. Chem. Phys. 15 (23) (2015) 1345313473. doi:10.5194/acp-15-13453-2015.

[37] E. Dieudonné, P. Chazette, F. Marnas, J. Totems, X. Shang, Lidar profiling of aerosol optical properties from paris to lake baikal (siberia), Atmos. Chem. Phys. 15 (9) (2015) 5007-5026. doi:10.5194/acp-15-5007-2015.

[38] G. Ancellet, J. Pelon, J. Totems, P. Chazette, A. Bazureau, M. Sicard, T. Di Iorio, F. Dulac, M. Mallet, Long-range transport and mixing of aerosol sources during the 2013 north american biomass burning episode: analysis of multiple lidar observations in the western mediterranean basin, Atmos. Chem. Phys. 16 (7) (2016) 4725-4742. doi:10.5194/ acp-16-4725-2016.

[39] E. Giannakaki, P. G. van Zyl, D. Müller, D. Balis, M. Komppula, Optical and microphysical characterization of aerosol layers over south africa by means of multi-wavelength depolarization and raman lidar measurements, Atmos. Chem. Phys. 16 (13) (2016) 8109-8123. doi:10.5194/ acp-16-8109-2016.

[40] H. Chang, T. T. Charalampopoulos, Determination of the wavelength dependence of refractive indices of flame soot, Proc. R. Soc. Lond. 430 (1880) (1990) 577-591. doi:10.1098/rspa.1990.0107.

[42] B. V. Scarnato, S. Vahidinia, D. T. Richard, T. W. Kirchstetter, Effects of internal mixing and aggregate morphology on optical properties of black 1020

[41] M. Hess, P. Koepke, I. Schult, Optical properties of aerosols and clouds: The software package opac, Bull. Amer. Meteor. Soc. 79 (5). doi:10.1175/ 1520-0477(1998) 079<0831:OPOAAC>2.0.C0;2. carbon using a discrete dipole approximation model, Atmos. Chem. Phys. 13 (10). doi:10.5194/acp-13-5089-2013. 
[43] J. Yon, A. Bescond, F. Liu, On the radiative properties of soot aggregates part 1: Necking and overlapping, J. Quant. Spectrosc. Radiat. Transfer 162 (Supplement C). doi:10.1016/j.jqsrt.2015.03.027.

[44] A. Brasil, T. Farias, M. Carvalho, A recipe for image characterization of fractal-like aggregates, J. Aerosol Sci. 30 (10) (1999) 1379-1389. doi: 10.1016/S0021-8502(99)00026-9.

[45] R. K. Chakrabarty, H. Moosmüller, L.-W. A. Chen, K. Lewis, W. P. Arnott, C. Mazzoleni, M. K. Dubey, C. E. Wold, W. M. Hao, S. M. Kreidenweis, Brown carbon in tar balls from smoldering biomass combustion, Atmos. Chem. Phys. 10 (2010) 6363-6370. doi:10.5194/acp-10-6363-2010.

[46] T. Nakayama, Y. Matsumi, K. Sato, T. Imamura, A. Yamazaki, A. Uchiyama, Laboratory studies on optical properties of secondary organic aerosols generated during the photooxidation of toluene and the ozonolysis of -pinene, J. Geophys. Res. 115 (D24) (2010) n/a-n/a, d24204. doi:10.1029/2010JD014387

[47] M. A. Yurkin, A. G. Hoekstra, The discrete-dipole-approximation code adda: Capabilities and known limitations, J. Quant. Spectrosc. Radiat. Transfer 112 (13). doi:10.1016/j.jqsrt.2011.01.031.

[48] M. Kahnert, Numerical solutions of the macroscopic Maxwell equations for scattering by non-spherical particles: A tutorial review, J. Quant. Spectrosc. Radiat. Transfer 178 (Supplement C) (2016) 22 - 37, electromagnetic and light scattering by nonspherical particles XV: Celebrating 150 years of Maxwell's electromagnetics. doi:10.1016/j.jqsrt.2015.10.029.

[49] A. Lakhtakia, G. W. Mulholland, On two numerical techniques for light scattering by dielectric agglomerated structures, J. Res. Natl. Inst. Stand. Technol. 98 (1993) 699-716. doi:10.6028/jres.098.046.

[50] M. Yurkin, A. Hoekstra, The discrete dipole approximation: An overview and recent developments, J. Quant. Spectrosc. Radiat. Transfer 106 (1) 
(2007) 558-589, iX Conference on Electromagnetic and Light Scattering by Non-Spherical Particles. doi:10.1016/j.jqsrt.2007.01.034.

[51] M. Kahnert, T. Nousiainen, H. Lindqvist, M. Ebert, Optical properties of light absorbing carbon aggregates mixed with sulfate: assessment of different model geometries for climate forcing calculations, Opt. Express 20 (2012) 10042-10058. doi:10.1364/OE.20.010042.

[52] K. Schmidt, M. Yurkin, M. Kahnert, A case study on the reciprocity in light scattering computations, Opt. Express 20 (2012) 23253-23274. doi: 10.1364/OE.20.023253.

[53] M. I. Mishchenko, J. W. Hovenier, Depolarization of light backscattered by randomly oriented nonspherical particles, Opt. Lett. 20 (12) (1995) 13561358. doi:10.1364/OL.20.001356.

[54] M. I. Mishchenko, K. Sassen, Depolarization of lidar returns by small ice crystals: An application to contrails, Geophys. Res. Lett. 25 (3) (1998) 309-312. doi:10.1029/97GL03764.

[55] M. Fiebig, A. Petzold, U. Wandinger, M. Wendisch, C. Kiemle, A. Stifter, M. Ebert, T. Rother, U. Leiterer, Optical closure for an aerosol column: Method, accuracy, and inferable properties applied to a biomass-burning aerosol and its radiative forcing, J. Geophys. Res. 107 (D21) (2002) LAC 12-1-LAC 12-15, 8130. doi:10.1029/2000JD000192.

[56] T. Murayama, D. Müller, K. Wada, A. Shimizu, M. Sekiguchi,

- T. Tsukamoto, Characterization of asian dust and siberian smoke with multi-wavelength raman lidar over tokyo, japan in spring 2003. Geophys. Res. Lett. 31 (23) (2004) n/a-n/a, 123103. doi:10.1029/2004GL021105. URL $10.1029 / 2004$ GL021105

[57] D. Müller, I. Mattis, U. Wandinger, A. Ansmann, D. Althausen, A. Stohl, Raman lidar observations of aged siberian and canadian forest fire smoke in the free troposphere over germany in 2003: Microphysical particle 
characterization, J. Geophys. Res. 110 (D17) (2005) n/a-n/a, d17201. doi:10.1029/2004JD005756

[61] F. Dahlkötter, M. Gysel, D. Sauer, A. Minikin, R. Baumann, P. Seifert, A. Ansmann, M. Fromm, C. Voigt, B. Weinzierl, The pagami creek smoke plume after long-range transport to the upper troposphere over europe - aerosol properties and black carbon mixing state, Atmos. Chem. Phys. 14 (12) (2014) 6111-6137. doi:10.5194/acp-14-6111-2014.

[62] M. Kahnert, Modelling the optical and radiative properties of freshly emitted light absorbing carbon within an atmospheric chemical trans1100 ㅁ port model, Atmos. Chem. Phys. 10 (3) (2010) 1403-1416. doi:10.5194/ acp-10-1403-2010.

[63] M. I. Mishchenko, J. M. Dlugach, L. Liu, Linear depolarization of lidar returns by aged smoke particles, Appl. Opt. 55 (35). doi:10.1364/A0.55. 009968 . 


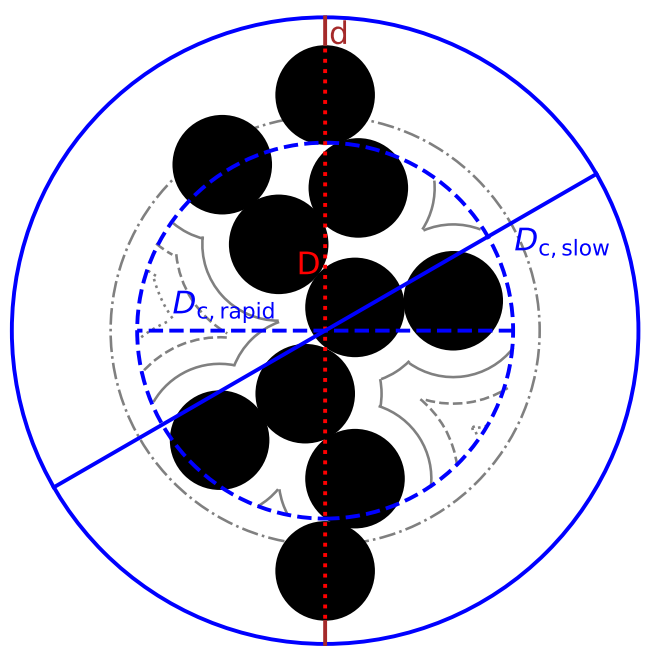

Figure 1: Illustration of the coating mechanism in the proposed coating models. D (red) denotes the maximum dimension of the aggregate, $d$ (orange) the thickness of a dipole layer. The critical sphere as defined in [26] and in the slow-transition model as well as the corresponding critical diameter $D_{\text {c,slow }}$ are shown in blue (solid line), the critical sphere as defined in the rapid-transition model and the corresponding diameter $D_{\text {c,rapid }}$ in blue (dashed line). The grey lines (solid, dashed, and dotted) illustrate the first three layers of coating that are added in the rapid-transition model. The grey dash-dotted line illustrates the first layer of coating that is being added after the critical sphere has been completely filled. 


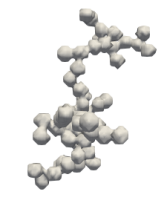

(a) $D_{f}=2.0$

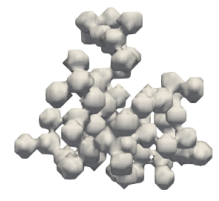

(b) $D_{f}=2.6$

Figure 2: Example of two bare aggregates with $N=64$ and $k_{0}=0.7$. a) aggregate with a fractal dimension of $\left.D_{f}=2.0 \mathrm{~b}\right)$ aggregate with a fractal dimension of $\left(D_{f}=2.6\right)$

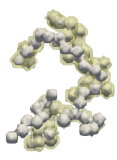

(a) $f_{\mathrm{vol}}=50 \%$

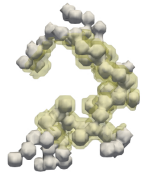

(d) $f_{\mathrm{vol}}=50 \%$

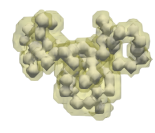

(b) $f_{\text {vol }}=25 \%$

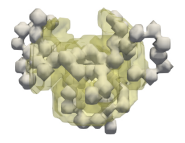

(e) $f_{\mathrm{vol}}=25 \%$

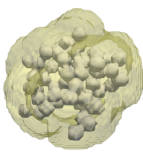

(c) $f_{\mathrm{vol}}=10 \%$

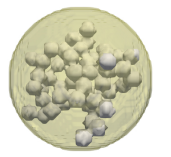

(f) $f_{\mathrm{vol}}=10 \%$

Figure 3: Examples of six coated aggregates with $N=64$ and $k_{0}=0.7$. The aggregate is shown in grey and the coating is shown in yellow. The top row (a-c) shows aggregates coated using the slow-transition model. In the bottom row (d-f) coated aggregates obtained with the rapid-transition model are shown. a, d) aggregate with $\left.f_{\mathrm{vol}}=50 \%\left(D_{f}=2.2\right) \mathrm{b}, \mathrm{e}\right)$ aggregate with $\left.f_{\mathrm{vol}}=25 \%\left(D_{f}=2.4\right) \mathrm{c}, \mathrm{f}\right)$ aggregate with $f_{\mathrm{vol}}=10 \%\left(D_{f}=2.6\right)$ 


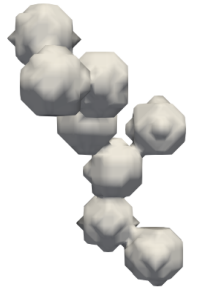

(a) $C_{o v}=0.0$

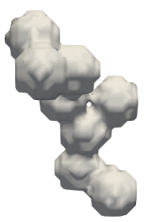

(b) $C_{o v}=0.15$

Figure 4: Example of two bare aggregates with $N=8$ and $D_{f}=2.0$. a) aggregate with pointcontacting monomers $\left.\left(C_{o v}=0.0\right) \mathrm{b}\right)$ aggregate with overlapping monomers $\left(C_{o v}=0.15\right)$
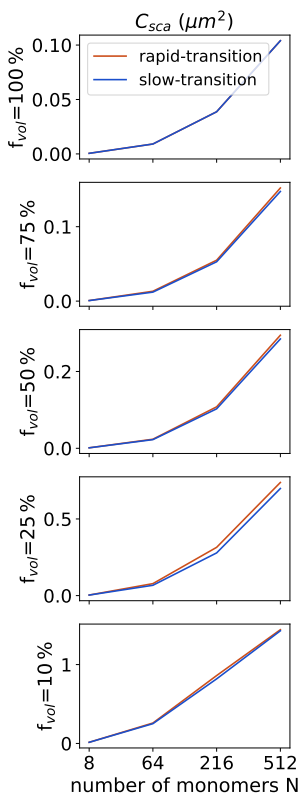
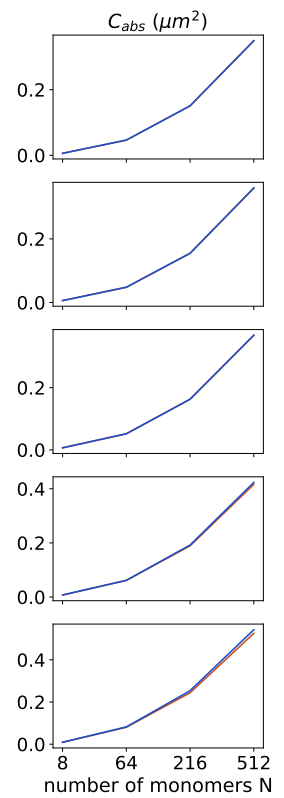
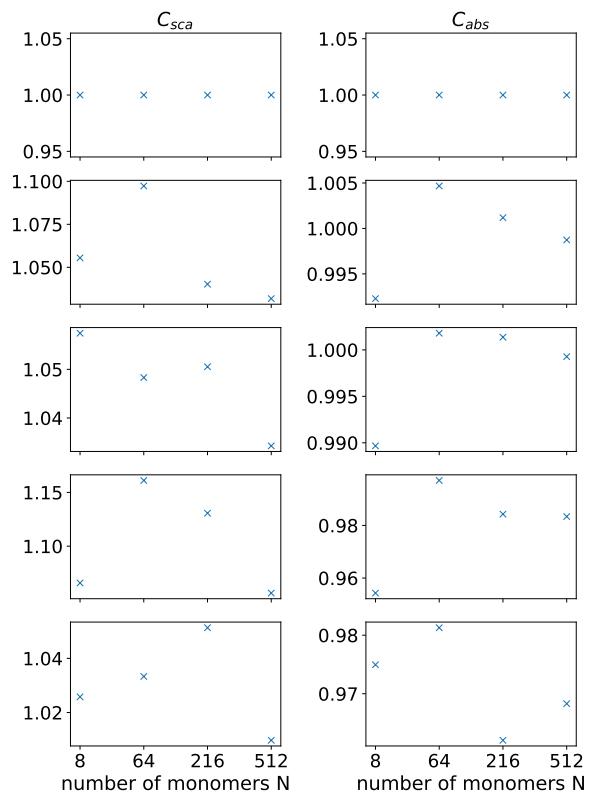
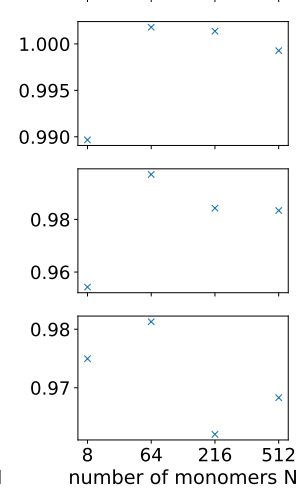

Figure 5: Comparison of optical cross sections for both coating models at $\lambda=532 \mathrm{~nm}$. The first column shows the scattering cross sections and the second column shows the absorption cross section for the rapid-transition model (red line) and the slow-transition model (blue line). The third column shows the ratio of the scattering cross section calculated for the rapid-transition and slow-transition model, and the fourth column shows the corresponding ratio for the absorption cross section. The rows correspond, from top to bottom, to soot volume fractions of $100 \%, 75 \%, 50 \%, 25 \%$, and $10 \%$, respectively. 

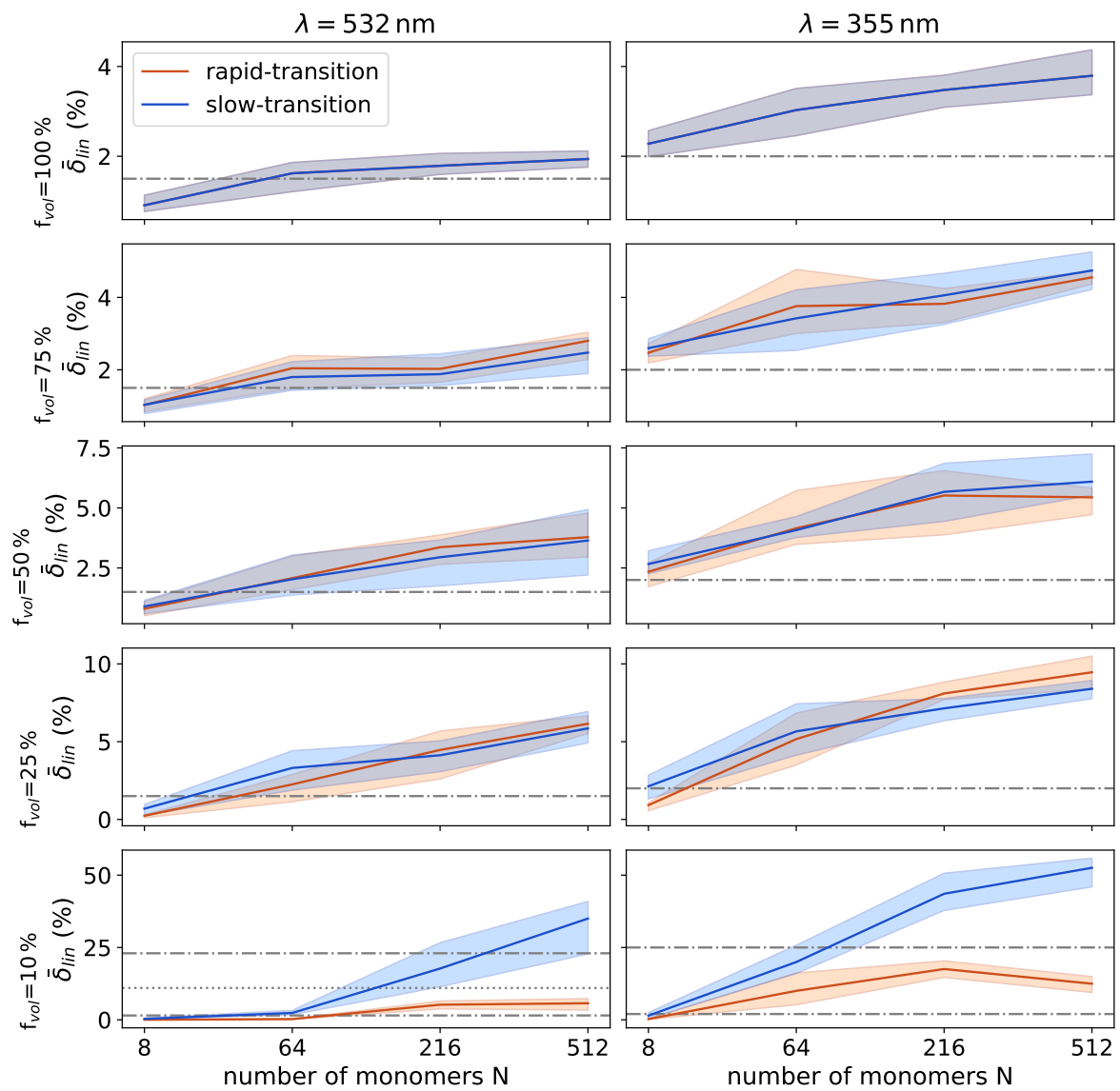

Figure 6: Linear backscattering depolarisation ratio at $\lambda=0.532 \mu \mathrm{m}$ (left colum) and at $\lambda=0.355 \mu \mathrm{m}$ (right column) for soot volume fractions $f_{\mathrm{vol}}=100 \%$ (first row), $75 \%$ (second row), $50 \%$ (third row), $25 \%$ (fourth row) and $10 \%$ (fifth row) computed for the rapid-transition (red) and the slow-transition model (blue). The dark lines correspond to the arithmetic mean of an ensemble of five different stochastic realisations of the aggregate geometry. The shaded area indicates the maximum variation within the ensemble. The grey dash-dotted lines indicate lower and upper bound of reported lidar field observations. Most reported values at $\lambda=0.532 \mu \mathrm{m}$ are below $11 \%$ (indicated by the grey dotted line). 

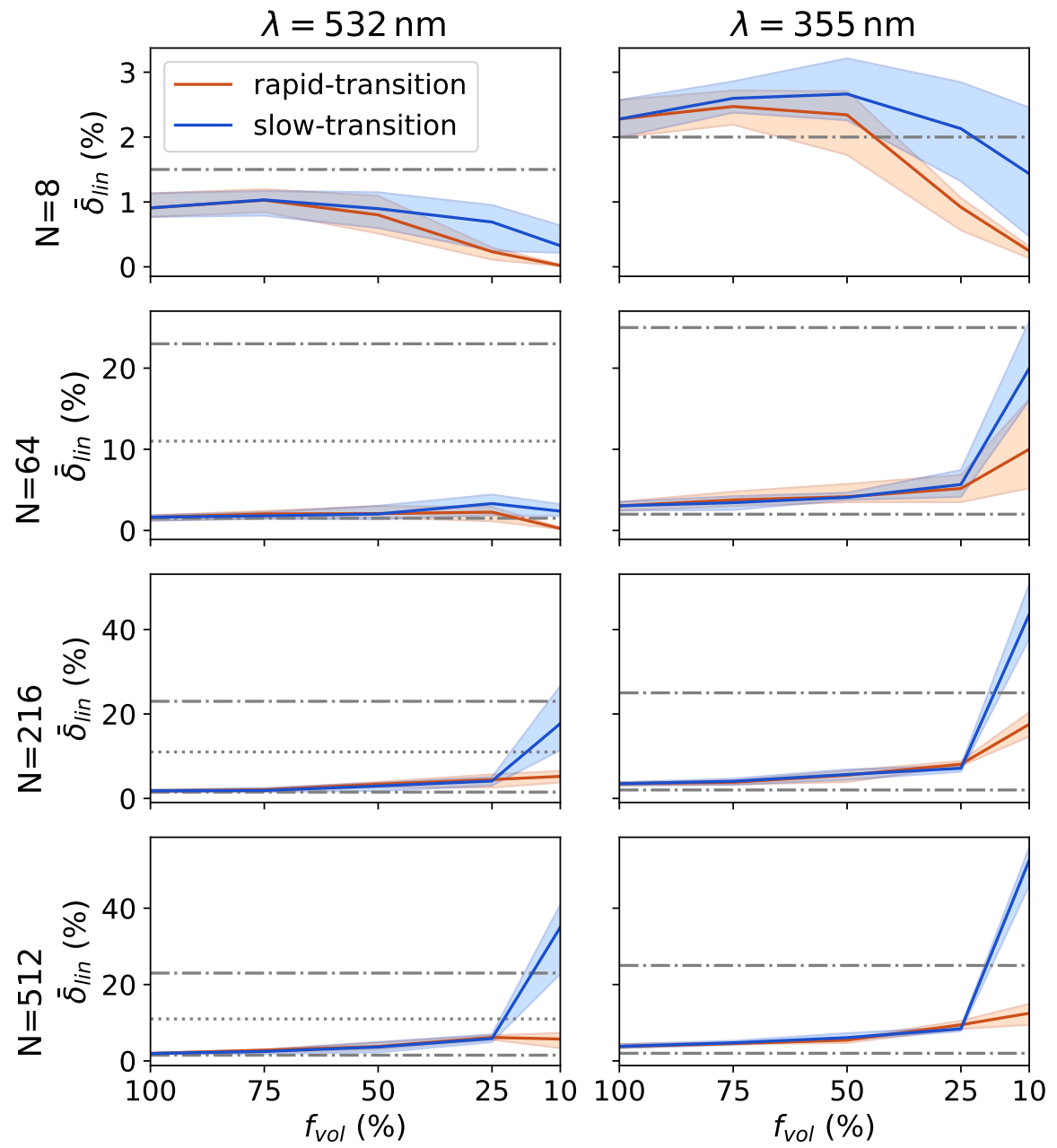

Figure 7: Linear backscattering depolarisation ratio at $\lambda=0.532 \mu \mathrm{m}$ (left colum) and at $\lambda=0.355 \mu \mathrm{m}$ (right column) for number of monomers $N=8$ (first row), $N=64$ (second row), $N=216$ (third row) and $N=512$ (forth row) computed for the rapid-transition (red) and the slow-transition model (blue). The lines and shadings are as in Fig. 6. 

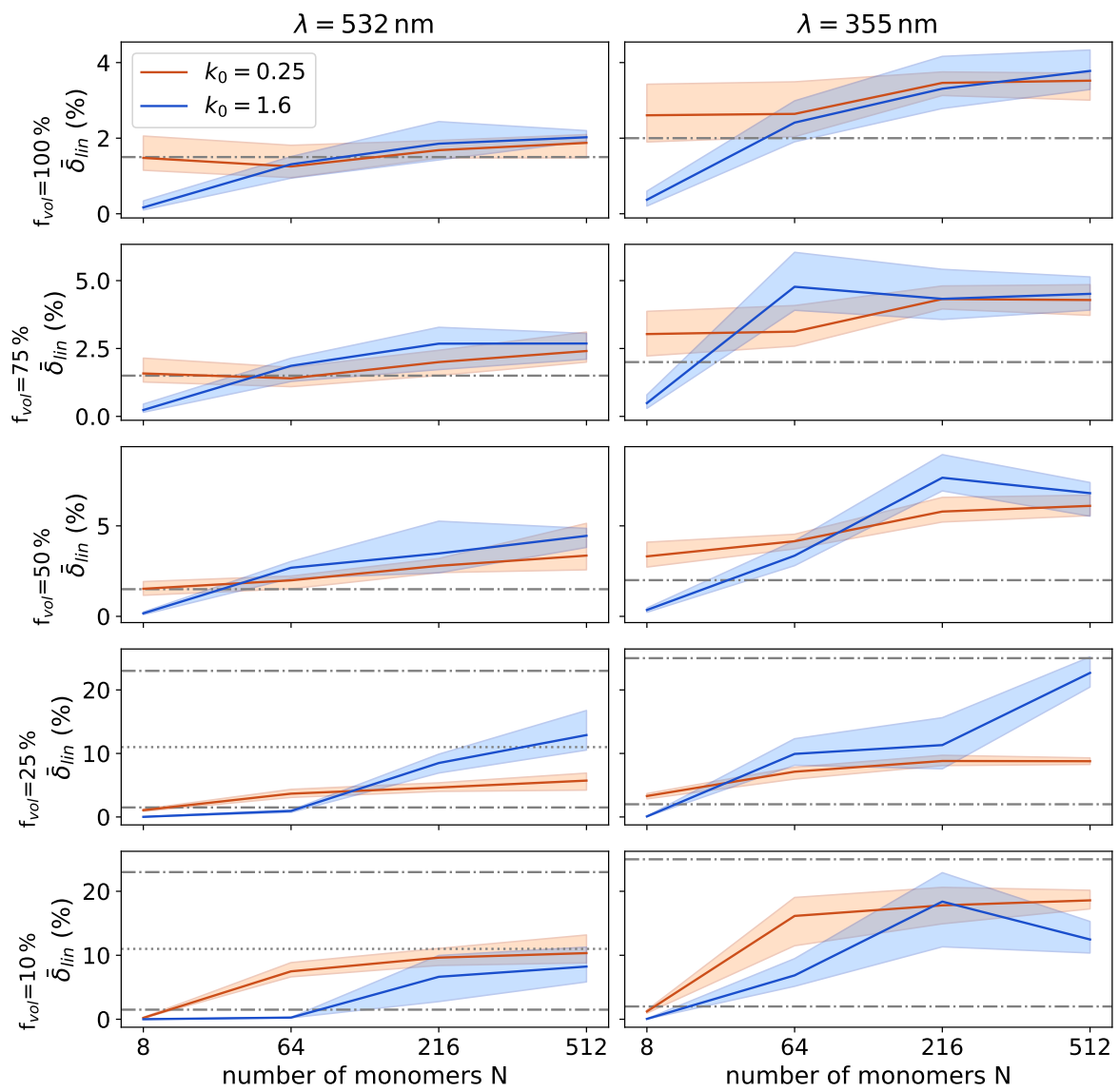

Figure 8: As Fig. 6 but for different fractal prefactors. The linear depolarisation ratios were calculated using the rapid transition model for two fractal prefactors $k_{0}=0.25$ (red) and $k_{0}=1.6$ (blue), which corresponds to the range reported in [29]. 

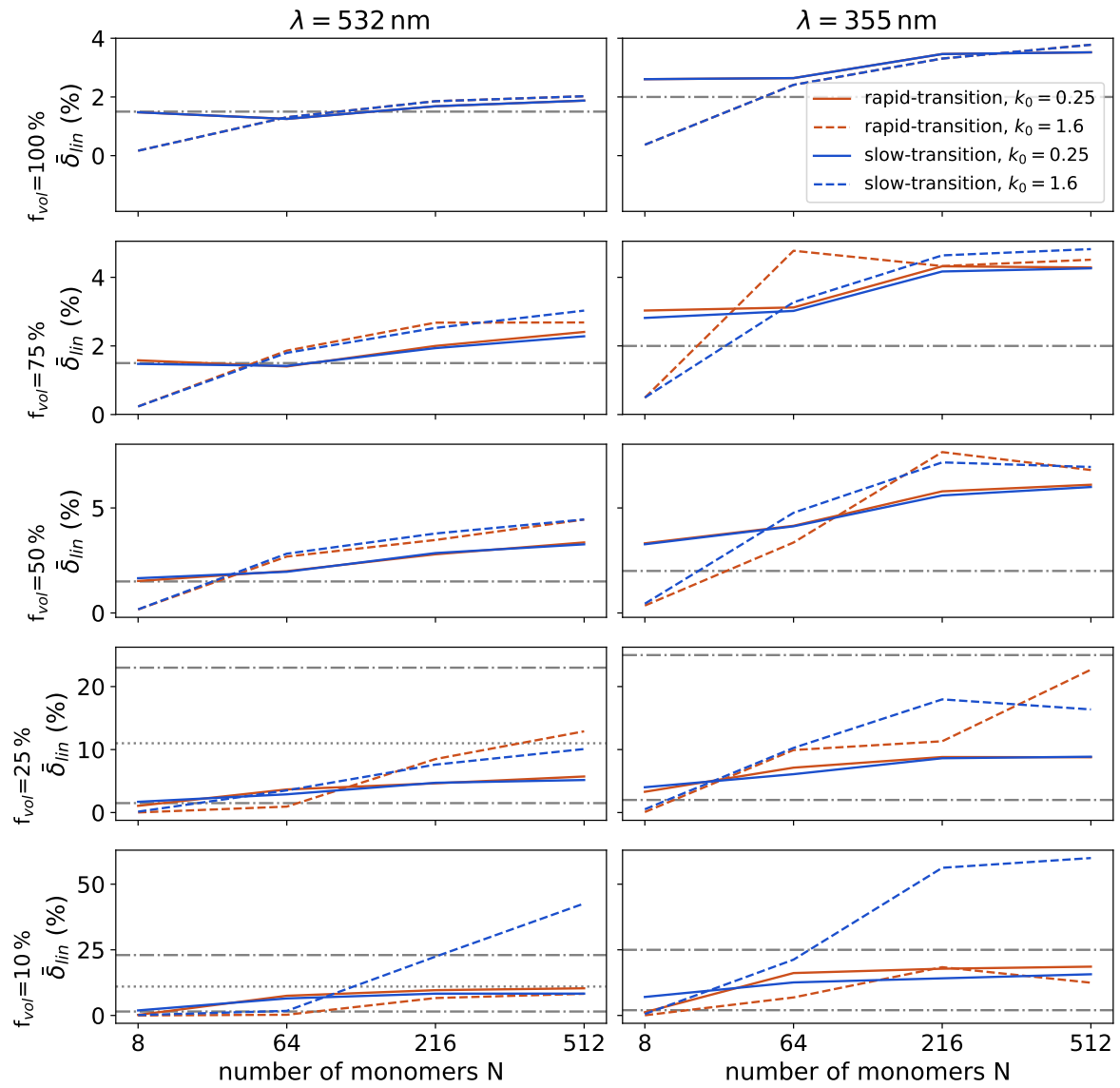

Figure 9: Linear backscattering depolarisation ratio at $\lambda=0.532 \mu \mathrm{m}$ (left colum) and at $\lambda=0.355 \mu \mathrm{m}$ (right column) for soot volume fractions $f_{\mathrm{vol}}=100 \%$ (first row), $75 \%$ (second row), $50 \%$ (third row), $25 \%$ (fourth row) and $10 \%$ (fifth row) computed for the rapid-transition (red) and the slow-transition model (blue) and for the two different fractal prefactors in 8 The solid lines indicate a fractal prefactor of $k_{0}=0.25$ and the dashed lines indicate a fractal prefactor of $k_{0}=1.6$. The grey lines are as in 6 

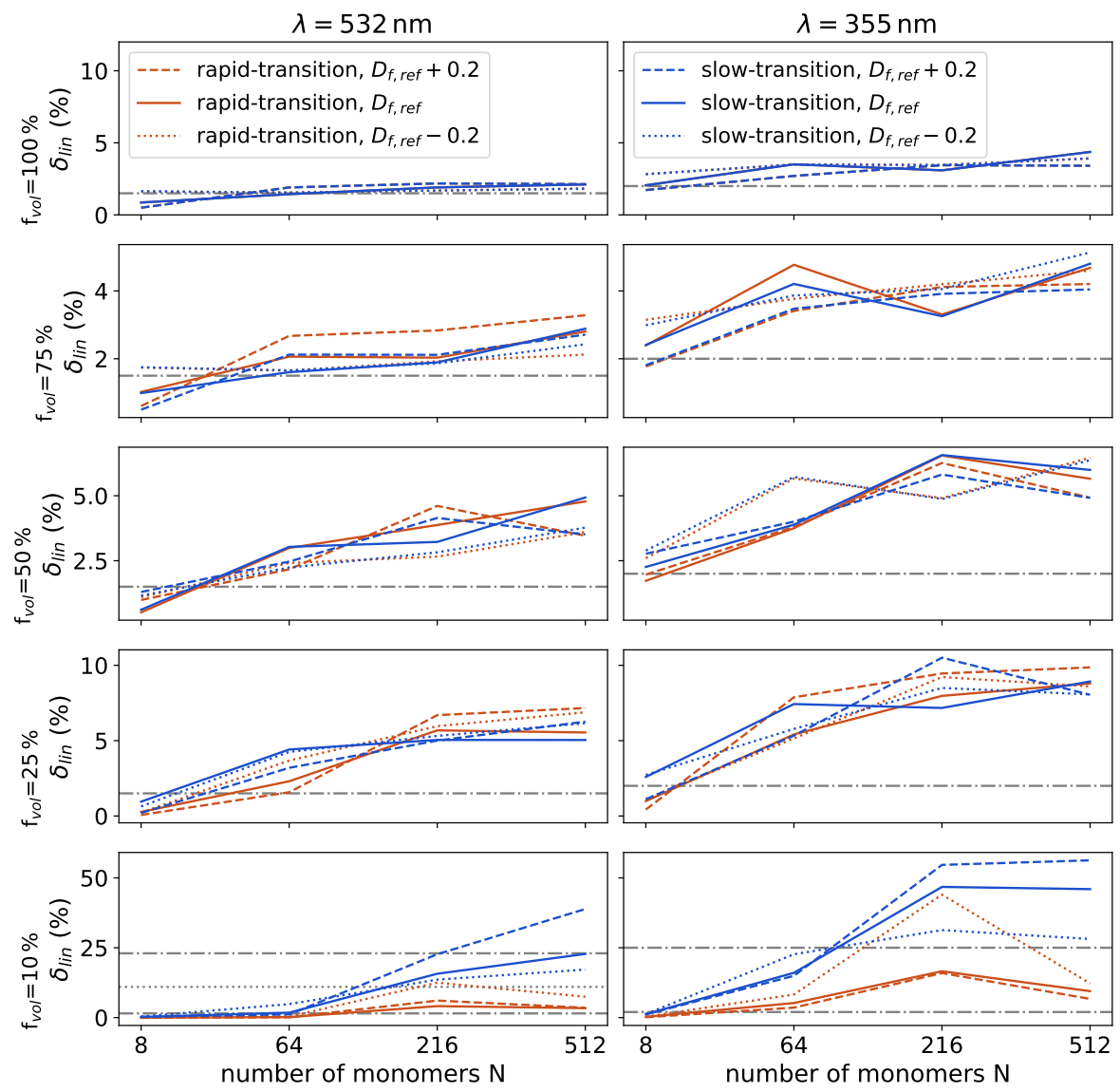

Figure 10: As Fig. 9 but for changes in fractal dimension. $D_{f, \text { ref }}$ (indicated by solid lines) refers to the values in Tab. 1 More compact aggregates $\left(D_{f, r e f}+0.2\right)$ are indicated by dashed lines. The dotted lines indicate lacier aggregates $\left(D_{f, r e f}-0.2\right)$. 

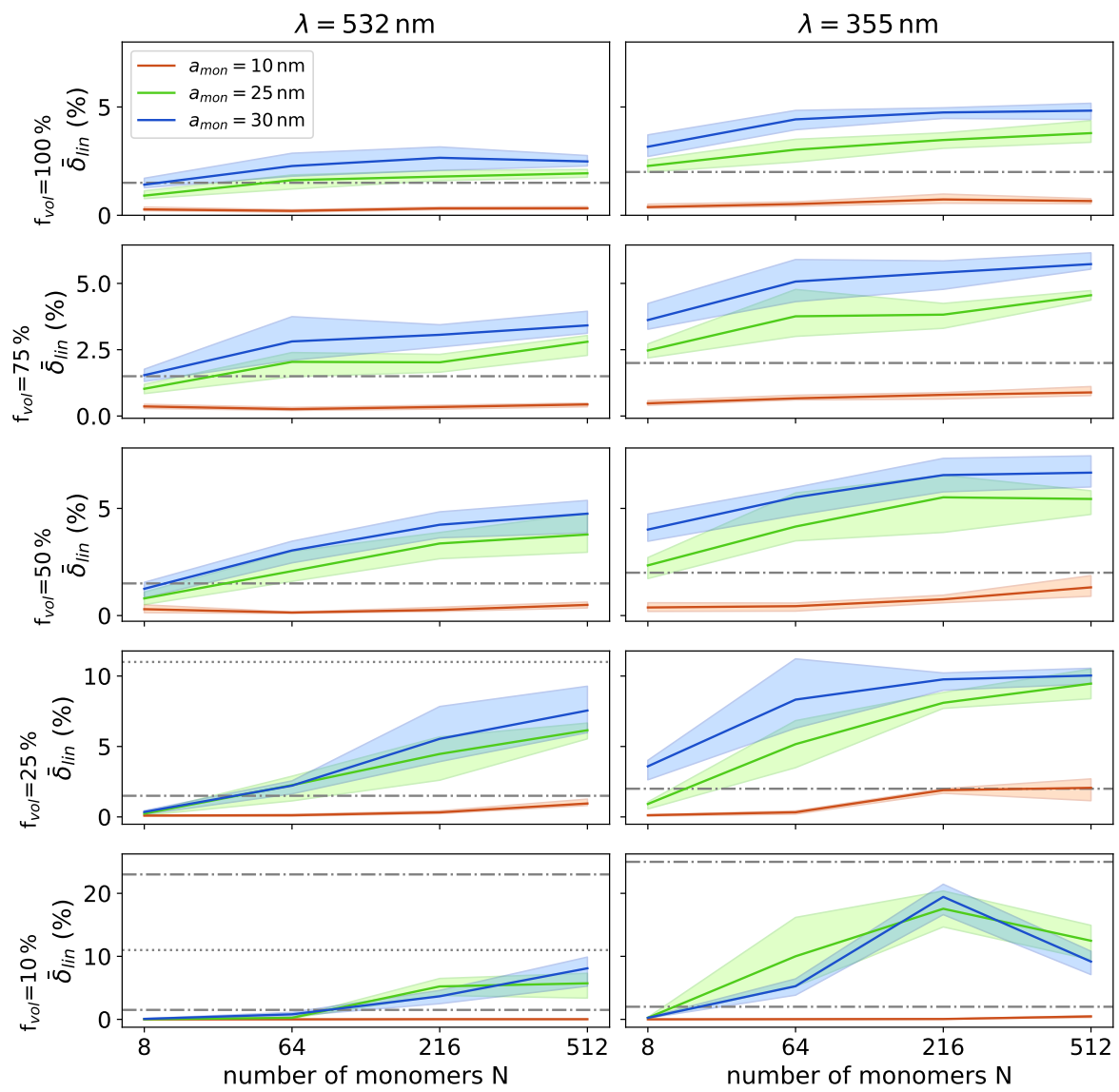

Figure 11: Linear backscattering depolarisation ratio for different monomer radii: $a=$ $0.015 \mu \mathrm{m}$ (red), $a=0.025 \mu \mathrm{m}$ (green) and $a=0.03 \mu \mathrm{m}$ (blue) using the rapid-transition model and assuming a constant number of monomers. The panels are as in Fig. 6 

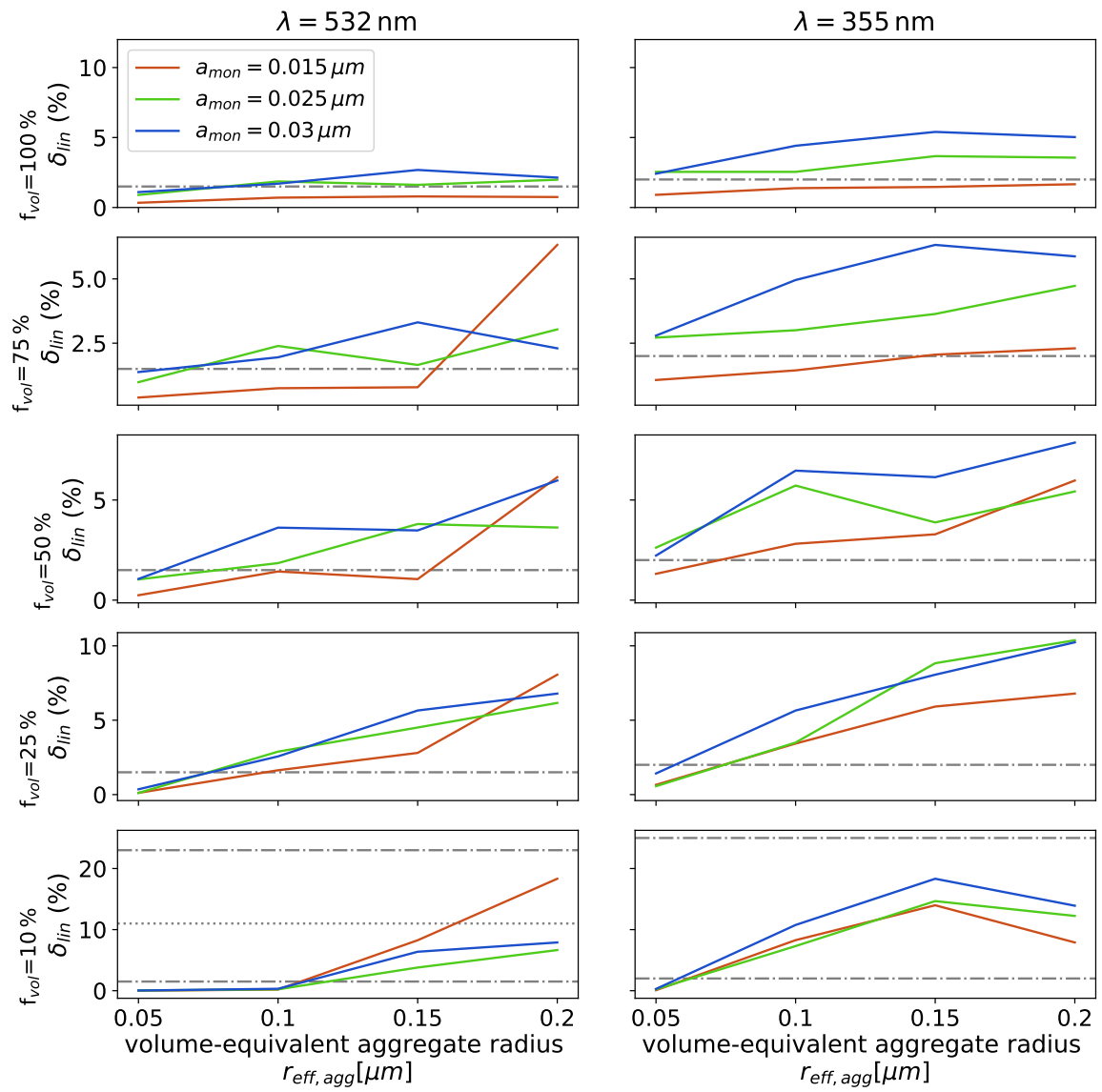

Figure 12: Linear backscattering depolarisation ratio for different monomer radii: $a=$ $0.015 \mu \mathrm{m}$ (red), $a=0.025 \mu \mathrm{m}$ (green) and $a=0.03 \mu \mathrm{m}$ (blue) using the rapid-transition model. The depolarisation ratios are shown as function of the volume-equivalent aggregate radius, which was kept constant for the different monomer radii. The panels are as in Fig. 6 

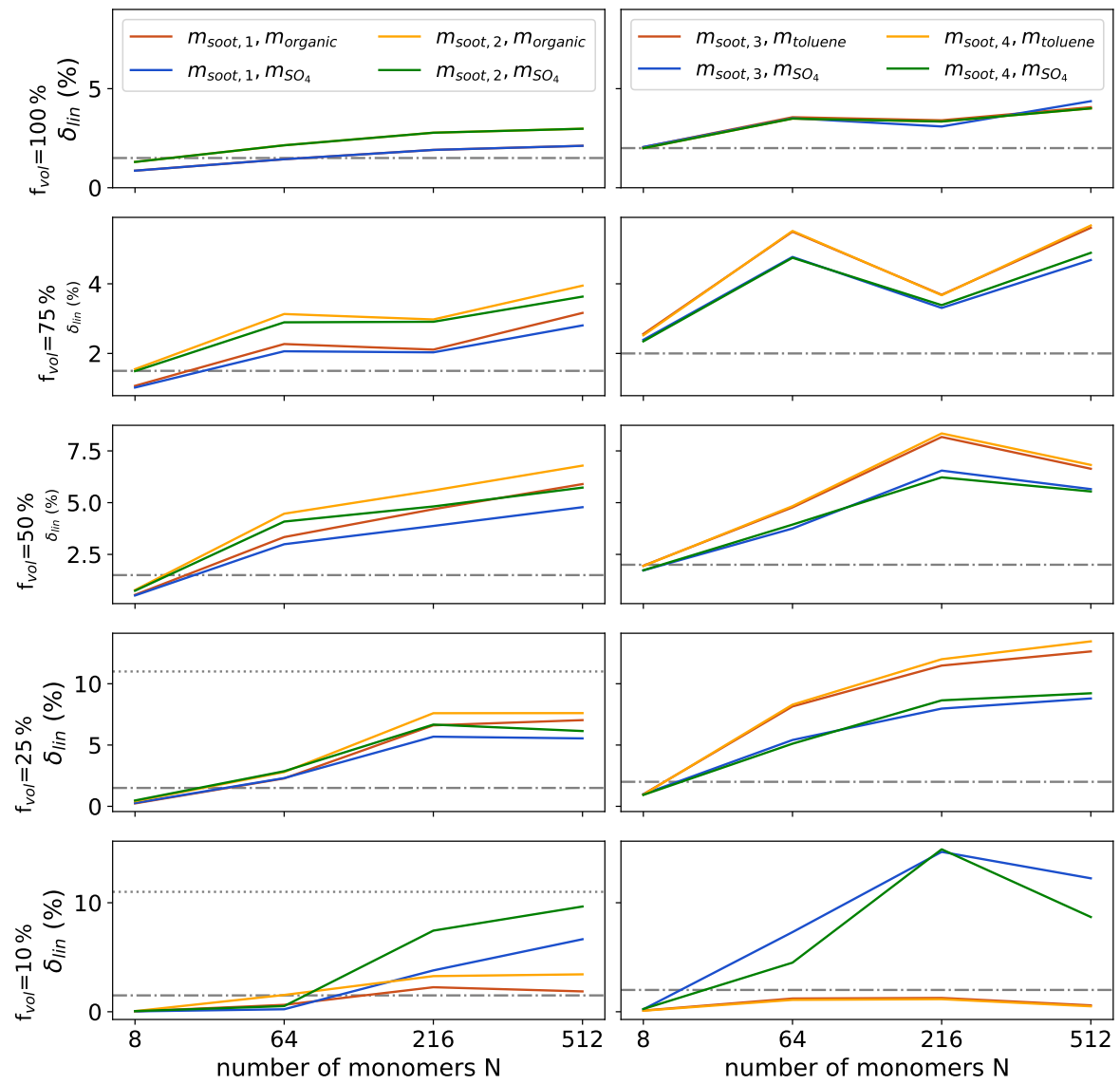

Figure 13: Linear backscattering depolarisation ratio as in Fig. 6. but showing results obtained with the rapid-transition model for a single geometry per aggregate size, and by using different combinations of the complex refractive index $m$ for the soot aggregate and the coating. The colours refer to the different combinations of refractive indices depending on the wavelength. For $\lambda=0.532 \mu \mathrm{m}$ (left column): $m_{\text {soot }, 1}=1.76+i 0.63, m_{\text {organic }}=1.53+i 0.0055$ (dark red), $m_{\text {soot }, 1}, m_{\mathrm{SO}_{4}}=1.43+i 10^{-8}$ (blue), $m_{\text {soot }, 2}=1.95+i 0.79, m_{\text {organic }}$ (orange) and $m_{\text {soot }, 2}, m_{\mathrm{SO}_{4}}$ (green). For $\lambda=0.355 \mu \mathrm{m}: m_{\text {soot }, 3}=1.66284+i 0.71528$ and $m_{\text {toluene }}=$ $1.632+i 0.047$ (dark red), $m_{\text {soot }, 4}=1.68586+i 0.67251$ and $m_{\text {toluene }}$ (orange), $m_{\text {soot }, 3}$ and $m_{\mathrm{SO}_{4}}=1.45+i 10^{-8}$ (blue) and $m_{\mathrm{soot}, 4}$ and $m_{\mathrm{SO}_{4}}$ (green). 

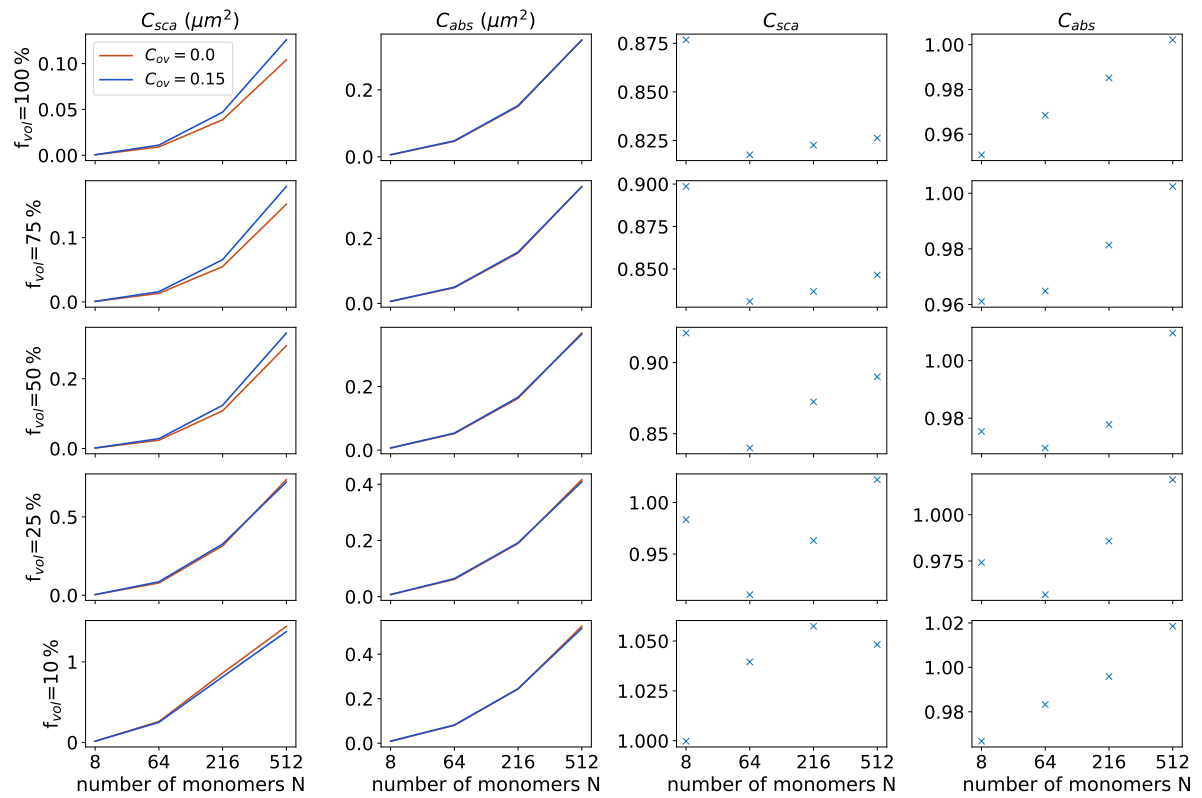

Figure 14: Comparison of optical cross sections for non-overlapping ( $C_{o v}=0.0$, red lines $)$ and overlapping ( $C_{o v}=0.15$, blue lines $)$ monomers at $\lambda=532 \mathrm{~nm}$ using the rapid-transition coating model. The rows are as in Fig. 5 The third column shows the ratio $C_{\mathrm{sca}}(0.0) / C_{\mathrm{sca}}(0.15)$ of the scattering cross sections, the fourth column shows the corresponding ratio for the absorption cross section. 

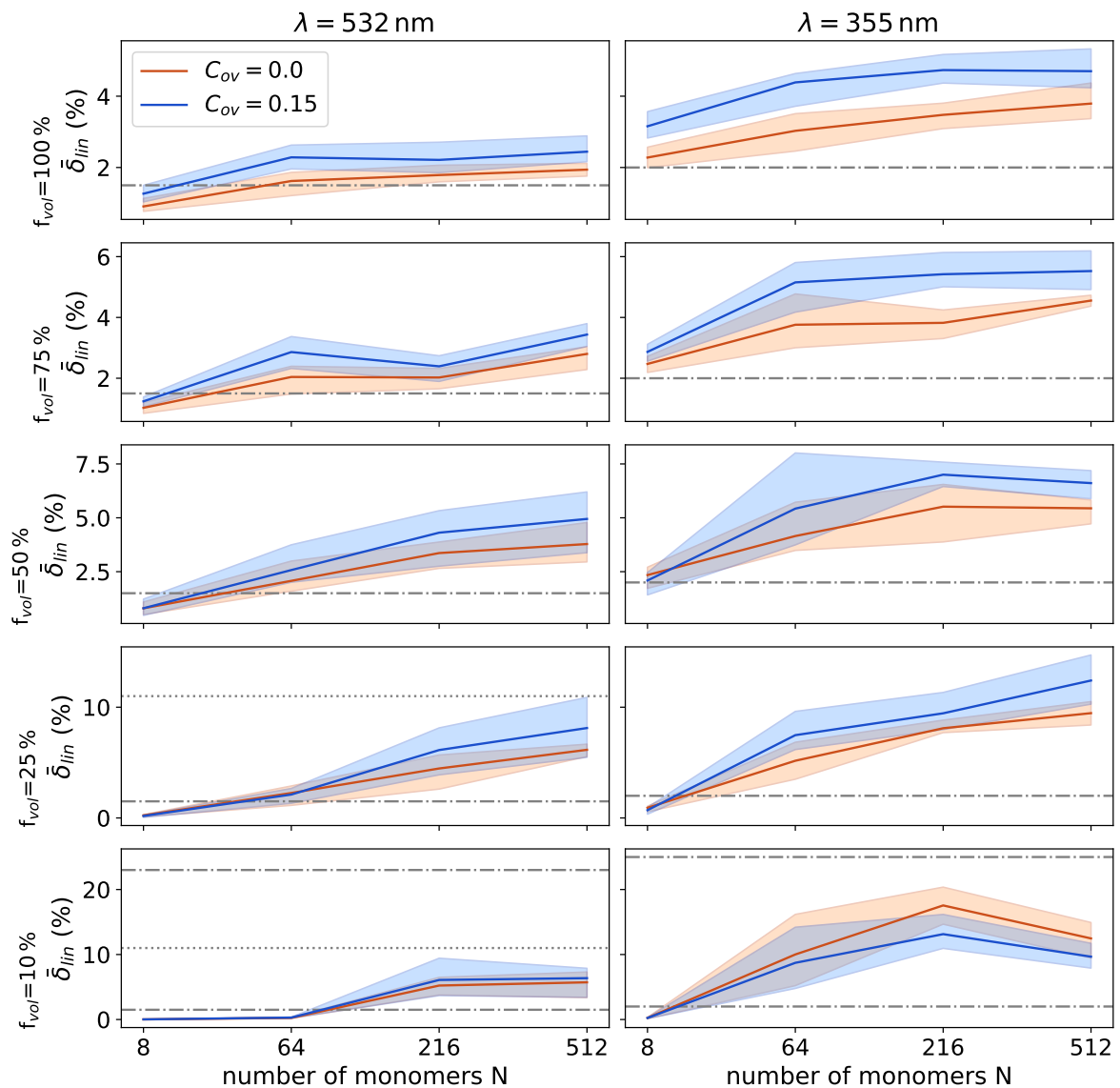

Figure 15: As Fig. 6 but comparing computations with the rapid-transition model in conjunction with an overlap factor of $C_{o v}=0.0$ (red) and $C_{o v}=0.15$ (blue). 